\title{
Ethnic Diversity and Well-Being*
}

\author{
Alpaslan Akay ${ }^{\dagger} \quad$ Amelie Constant ${ }^{\ddagger}$ Corrado Giulietti ${ }^{\S} \quad$ Martin Guzi $\mathbb{I}^{-}$ \\ September 6, 2016
}

(forthcoming in the Journal of Population Economics, 2016)

Accepted version: September 2016

\begin{abstract}
This paper investigates how ethnic diversity, measured by immigrants' nationalities, influences the well-being of the host country. Using panel data from Germany from 1998 to 2012, we find a positive effect of ethnic diversity on the well-being of German natives. Our finding is robust to alternative definitions of ethnic diversity and to the non-random selection of natives and immigrants into regions. The positive effect of ethnic diversity is stronger for immigrant groups that are culturally and economically closer to Germany. Consistent with this result, we document the existence of two mechanisms explaining the influence of ethnic diversity on well-being: productivity as captured by immigrants' skills and assimilation - and social capital - particularly in relation to the creation of a multicultural environment.

JEL codes: C90, D63, J61

Keywords: Ethnic diversity, subjective well-being, assimilation, multiculturality.
\end{abstract}

${ }^{*}$ We are grateful to two anonymous referees and the Editor Klaus F. Zimmermann for constructive feedback. We thank Peter Huber, Ruud J. A. Muffels, Jackie Wahba, and participants to the seminar at Beijing Normal University, IOS Regensburg, Reflect at Tilburg University and WIFO in Vienna for their useful comments. We are also indebted to Georgios Tassoukis for helping us collecting data from the German Federal Statistical Office and the statistical offices of the Länder.

†University of Gothenburg - alpaslan.akay@gu.se

${ }_{\ddagger}^{\ddagger}$ Princeton University - afconstant299@gmail.com

§University of Southampton - c.giulietti@soton.ac.uk

IMasaryk University - martin.guzi@econ.muni.cz 


\section{Introduction}

As migration from developing to developed regions continues to rise, diversity in the host countries, observed in terms of ethnicity, language, culture, religion and gender is substantially increasing. Along with other social scientists, economists have also expressed interest in understanding the impact of immigration and ethnic diversity on the social, economic, and political outcomes of the host society (e.g., Alesina et al., 1999, Ottaviano and Peri, 2005, Glitz, 2014). Recent works suggest that there are short and long-run effects of ethnic diversity on several outcomes of both natives and immigrants. On the one hand, and mostly in the US, ethnic diversity is negatively correlated with social capital or cohesion measured by trust, altruism, reciprocity, cooperation and civic engagement (e.g., Portes, 1998, Alesina et al., 1999, Alesina and La Ferrara, 2002, Putnam, 2007). On the other hand, other studies found that ethnic diversity is either not negatively related to social trust, or it is even positively correlated to it (e.g., Kazemipur, 2006 for Canada, Sturgis et al., 2011 and Sturgis et al., 2014 for the UK, and Stolle et al., 2013 for Germany). Moreover, some studies found positive effects of ethnic diversity on the labor market outcomes of both natives and immigrants through gains in productivity (Ottaviano and Peri, 2005, 2006, Trax et al., 2015, Suedekum et al., 2014, Glitz, 2014) as well as increases in innovation (Hewlett et al., 2013). However, no study so far has examined the impact of ethnic diversity directly on the welfare of natives. This paper fills this gap in the literature by investigating how ethnic diversity in Germany influences the utility of natives using subjective well-being (SWB) as a proxy for the experienced utility (Frey and Stutzer, 2002, Kahneman and Sugden, 2005).

Germany serves as an important case study for several reasons. First, it is a high immigration country, with immigrants coming from nearly every country in the world. According to figures from the German Federal Statistical Office, there were 7.5 million foreigners at the end of 2014, making up about $9.3 \%$ of the total population in Germany. ${ }^{1}$ The ethnic composition of immigrants changed substantially in the past years and in an uneven manner across the regions of Germany. This is the result of several factors, including the immigration of guest workers in the 1960s and 1970s (many immigrants decided to permanently settle in Germany), the immigration of ethnic Germans (i.e., individuals of German descent born abroad who moved to Germany), the increased labor mobility within the European Union (the transition rules imposed after the 2004 EU enlargement were lifted in 2011), and more recently the waves of refugees and asylum seekers.

\footnotetext{
${ }^{1}$ http://www.statistik-portal.de/Statistik-Portal/en/en_jb01_jahrtab2.asp. Last access: September $5^{\text {th }}$ 2016. The number of individuals with "immigration background" is much higher, and was approximately 16.4 million, representing about $20.3 \%$ of the population (according to the 2014 microcensus). The majority of people with a migrant background $(56 \%)$ have also German passport.
} 
A second important reason of focusing on Germany is that it is home to the German SocioEconomic Panel (GSOEP), one of the largest and longest-running longitudinal datasets. The availability of rich and nationally representative panel data is crucial to our analysis, as it allows us to control for various sources of unobserved heterogeneity. Also, we are able to match GSOEP with data from the Central Registry of Foreigners - which provides the exact counts of individuals living in a locality by country of nationality. This allows a precise and detailed measurement of ethnic diversity.

Some studies using firm data from German regions suggest that natives achieve higher productivity levels when the workforce is more ethnically diverse. Suedekum et al. (2014) use administrative records with information on wages, employment and the nationality of immigrants aggregated at the level of 326 West Germany counties over the period 1996-2005. They find a strong positive effect of immigrant diversity on both the regional wage and the employment rates of native workers. Using the same dataset at the establishment level in the manufacturing and service sectors during the period 1999-2008, Trax et al. (2015) show that the number of immigrants in the plant or in the region has no significant impact on plant's productivity. However, the authors report a positive association between the ethnic diversity of employees - measured by their nationality - and the productivity in the manufacturing sector.

Brunow and Blien (2014) explore a potential channel through which the productivity gains induced by the ethnic diversity of employees are realized. Using German establishment level data, the authors show that, for a given level of revenues, firms with ethnically diverse workforce employ fewer workers. Their suggested explanation is that a culturally diverse work environment produces interactions and positive externalities, and thus relatively less labor is needed. Based on the same data, Brunow and Nijkamp (2012) show that a culturally diverse skilled workforce provides a productivity advantage to establishments and increases their market size. Further tests reveal, however, that the diversity of low-skilled workers has no effect on productivity.

To the best of our knowledge, this is the first paper that investigates the effect of ethnic diversity on the well-being of natives. ${ }^{2}$ Our analysis complements the evidence of Akay et al. (2014), who discover a positive effect of the immigrant share in the region on the well-being of natives. Since migration is a complex phenomenon and can affect domains beyond those usually researched - e.g., labor market outcomes - our approach allows us to provide insight on how both the size and the composition of migration affect overall Germans' utility.

The identification strategy exploits individual longitudinal data that are matched to the

\footnotetext{
${ }^{2}$ We focus on the subjective well-being of individuals who report being born in Germany. We use the term Germans and natives interchangeably.
} 
exact counts of immigrants in 96 German regions over the period 1998-2012. We measure ethnic diversity with an index constructed using up to 174 different nationalities of immigrants. Nationality data are obtained from population registers, and have thus the advantage of being virtually free from measurement error issues. To investigate the relationship of interest, we first estimate several SWB equations in which, besides the key ethnic diversity index, we control for individual observed characteristics, as well as for regional and individual unobserved heterogeneity that could be correlated with observables. The results from our fixed-effects specification suggest that ethnic diversity positively affects the well-being of natives. This result is robust to alternative econometric specifications and definitions of ethnic diversity. Adopting the approach of Alesina et al. (2016), we document that the effect of ethnic diversity is stronger when more weight is given to immigrant groups that are culturally and economically closer to Germany.

Although our econometric strategy is helpful to mitigate the confounding role of unobserved heterogeneity, we pay particular attention to possible threats to a causal interpretation of our results. In particular, we explore the role of non-random sorting of immigrants due to their self-selection into regions that have higher ethnic diversity (e.g., ethnic enclaves). We also analyze whether the internal migration of natives across regions occurs in response to higher diversity, in which case our results would suffer from selectivity bias. Overall, we find that our estimates are not affected by internal migration.

Finally, we investigate the potential mechanisms that could explain our results. We document that productivity and social capital are likely channels through which ethnic diversity influences well-being. The role of productivity emerges particularly in relation to immigrants' skills and assimilation, as the effect of ethnic diversity on Germans' well-being is stronger in regions where immigrants have better German language skills and feel closer to the German culture. The role of social capital is associated with the multicultural environment brought by ethnic diversity.

The remainder of the paper is organized as follows: in Section 2 we present the data and the measures of ethnic diversity. Section 3 outlines the econometric strategy. Section 4 presents the baseline results, along with various robustness checks and the heterogeneity analysis. Section 5 discusses the channels behind our results and Section 6 concludes.

\section{Data}

\subsection{Sample Selection}

Our main data source is the German Socio-Economic Panel (GSOEP), a large and nationally representative longitudinal dataset providing rich information on individual and household 
characteristics. GSOEP is a dataset widely used in the SWB literature (e.g., Winkelmann and Winkelmann, 1998, van Praag et al., 2003, Ferrer-i Carbonell, 2005, Akay et al., 2016), as well as in the migration literature (e.g., Constant and Massey, 2003, Jaeger et al., 2010, Akay et al., 2014). The data collection of GSOEP started in 1984 in West Germany, with a sample size of more than 25,000 individuals. The survey was subsequently extended to the whole Germany in 1990. The dataset has information on education, health, labor markets and income, as well as several SWB measures. One important aspect of GSOEP is the low attrition rate, which is a crucial aspect for our identification strategy.

The SWB measure that we employ is based on the question about life-satisfaction "How satisfied are you with your life as a whole, all things considered?". Answers are coded on an 11-point scale (0 stands for "completely dissatisfied" and 10 for "completely satisfied"). After decades of research, there is consensus that such measure is a good proxy for the SWB, and that it is also highly correlated with other measures used in the literature such as happiness and mental health (e.g., Clark and Oswald, 1994, Kahneman and Sugden, 2005). Since we are interested in the effect of diversity on natives' well-being, we restrict our sample to individuals who report being born in Germany and are aged between 16 and 64 years. It is important to point out that immigrants' children who are born in Germany but have not yet acquired citizenship are considered foreigners. ${ }^{3}$ We restrict our sample to the period 1998-2012 since the regional immigration data that we combine with the GSOEP are only available for these years. Lastly, we eliminate a few observations with missing values, obtaining a final sample of 188,123 individual $\times$ year observations.

\subsection{Regional Data}

The GSOEP contains information on the 96 "regional policy regions" of residence of individuals. The Raumordnungsregion (henceforth RORs) are self-contained regional units defined on the basis of economic characteristics and labor markets attributes (Knies and Spiess, 2007). The availability of spatial information allows us to link the individual data to ROR-level statistics from the Central Register of Foreign Nationals (Ausländerzentralregister, henceforth AZR) and from the Indikatoren und Karten zur Raum- und Stadtentwicklung (INKAR). From the AZR we obtain country of nationality information for the 404 districts of Germany (Kreise), which we aggregate at the ROR level. ${ }^{4}$ In some of our analyses, we also exploit the nationality data at the district level. The AZR provides the exact counts of

\footnotetext{
${ }^{3}$ We use the term foreigner interchangeably with immigrant.

${ }^{4}$ Kreise are administrative units that are self-contained within RORs. In rare cases there were small changes in the geography of Kreise, with some of them being classified in different RORs over time. We were able to match Kreise to the correct ROR thanks to lookup files provided with the data.
} 
foreigners for up to 174 nationalities in each district. ${ }^{5}$ These data are the basis to construct the ethnic diversity index, our key explanatory variable. The main advantage of AZR is that it provides an accurate and updated count of all registered immigrants by nationality. ${ }^{6}$ From INKAR we extracted regional indicators for the 96 RORs. These include the immigrant share (i.e., the ratio between the stock of immigrants and the resident population), the male unemployment rate, and the value of the gross domestic product. These data are used in our regressions to control for region-specific time-variant confounders. ${ }^{7}$

\subsection{Measuring Ethnic Diversity}

Ethnic diversity can be gauged using several measures that capture dimensions such as concentration, entropy and segregation (Massey and Denton, 1988). Furthermore, ethnicity could be measured using different definitions, such as country of birth, nationality, ethnic origin, and ethnic self-identification. To be consistent with the definitions in our data sources, we opted for an ethnic diversity index (ED) constructed using information on the shares of immigrants from different nationalities living in each region. Our diversity measure is based on the Herfindahl-Hirschman index and has been used in many studies about the effects of ethnic fractionalization (see, e.g., Alesina et al., 2016, Trax et al., 2015). At every point in time, the ED index is calculated as follows:

$$
E D_{r}=1-\sum_{g}\left(\frac{m_{g r}}{m_{r}}\right)^{2},
$$

where $m_{g r}$ is the number of immigrants of nationality $g$ in region $r$ (in our case ROR) and $m_{r}$ is the total number of immigrants in each region. The index ranges between 0 and 1 and increases with both the number of groups and the evenness of the distribution of individuals across groups. It approaches 1 when an immigrant population in the region is composed by a large number of groups of relatively equal size and different origins. ${ }^{8}$ Note, our index

\footnotetext{
${ }^{5}$ Note that up to 2007 included data were supplied by the statistical offices of each State (Länd), but since 2008 data come from the German Federal Statistical Office. The major implication is that the number of nationalities available is different across the 16 States for the first period, while it is homogeneous for the period when federal-level data are used. The robustness checks presented in Section 4 show that the different number of available nationalities does not influence our results.

${ }^{6}$ AZR does not collect data on country of birth or on ethnicity; hence it is not possible to construct a diversity index based on these alternative dimensions. However, in our robustness analysis, we provide sensitivity checks around the definition of our index.

${ }^{7}$ At the time of writing, AZR data are available until 2014, while INKAR until 2012, hence we restrict our analysis up to this year. AZR data for the State Saxony-Anhalt are only available from year 2007.

${ }^{8}$ Note that the argument of the sum operator can also be represented as: $\frac{m_{g_{r}}}{m_{r}}=\left(\frac{m_{g r}}{m_{r}} / \frac{m_{g}}{m}\right) \times \frac{m_{g}}{m}$. The first component in brackets measures the spatial distribution of immigrants and in particular whether immigrants of a certain nationality are over- or under-represented (values above and below 1, respectively)
} 
excludes individuals with German citizenship. There are two reasons behind this choice. First, our aim is to investigate how the diversity "within" the immigrant population and not "with respect to natives" affects the well-being of German nationals. Second, in all our regressions, we control also for the immigrant share, which accounts already - by definition - for the Germans' population size.

Our index differs from other concepts employed in the literature to measure ethnic diversity. For example, it would only partially overlap with a birthplace diversity measure such as the one used by Alesina et al. (2016), which is based on countries of origin. ${ }^{9}$ We would also expect some differences if diversity would be measured using a dimension such as race. Since official statistics do not collect data by race, we are not able to test how different such index would be from ours. Yet, in one of our robustness checks in Table 3, we define our index using broad areas of origin, which are thought to proxy for racial differences. Finally, our diversity measure differs also from the concept of ethnic identity used by Constant and Zimmermann (2008) and Constant et al. (2009). In those works, the authors capture the identity of immigrants by using the "ethnosizer", an index which combines and weighs five elements of an immigrant's identity vis-à-vis the home and host country: language, culture, ethnic self-identification, ethnic interaction, and immigration history. Indeed, it is not possible to build measures of ethnic identity using the population registers data. However, to get an idea about the potential role of ethnic identity in our context, in Table 6 we investigate how the ethnic diversity index interacts with some of the components of the ethnosizer, namely language skills, identity assimilation and economic integration of immigrants.

in a region. This is sometimes referred to as the relative clustering index (see e.g., Borjas, 2000). The second component is the share of immigrants over the total number of immigrants in Germany and captures the relative size of each nationality group.

${ }^{9} \mathrm{~A}$ diversity index based on birthplace would differ from one based on nationality because of citizenship dynamics. If no immigrants naturalize, then there would be virtually no difference since country of birth would coincide with nationality. If one considers the extreme example of immigrants coming from a small country who all naturalize, then the number of immigrant groups would be different since in this hypothetical case one particular nationality would disappear. To understand exactly how the two indices would differ, one would need to either obtain exact counts of immigrants by birthplace (which to our knowledge do not exist at the same level of our nationality data) or to carefully account for citizenship dynamics (which is hard to do even with a good dataset like the GSOEP). To provide a rough idea of the potential differences between the two indices, we have calculated an index based on ethnic diversity (i.e., using reported nationality) and one based on birthplace diversity (i.e., using reported country of origin) based on pooled data from the GSOEP over the period of our analysis. The ED index is constructed like the one used in the paper but uses GSOEP instead of data from population registers. The birthplace diversity index is constructed in the same manner but uses information on the country of origin. After calculating the two indices, we note that for the same sample there are 120 reported countries of origin and 104 nationalities. This is likely due to some small group transiting into German citizenship. Not surprisingly - since it uses a larger number of groups the birthplace diversity index is slightly higher (0.91) than the ED index (0.85). While we recognize that it is difficult to speculate how our results would be had we had the possibility of using a birthplace diversity index, we would expect them not to be substantially different than those obtained with our ED index. 
While our main analysis is based on the ED index as defined above, we also explore alternative definitions and measures. First, by exploiting data at the district level, we derive the Shannon Entropy index. While related to the Herfindahl-Hirschman index, the Shannon Entropy index has the key advantage that it can be decomposed in two parts, a "within" component, which captures the average ethnic diversity within each ROR and a "between" component, which measures the level of spatial segregation of ethnic groups across districts. Second, in line with the approach of Alesina et al. (2016), we "augment" our ED index by weighting the various nationality groups according to their genetic/cultural and economic distance from Germany. Finally, in our robustness checks we consider indices of ethnic diversity constructed using different aggregations of the nationality groups.

\subsection{Ethnic Diversity in Germany}

Nowadays, Germany hosts immigrants from almost every country in the world and is one of the principal immigration destinations in the developed world. The history of large immigration in Germany dates back to the 1960s, when many foreign nationals immigrated under the so-called "guest-worker program". The program was introduced as a solution to the substantial labor shortages that Germany encountered during its post-World War II expansion era. Major sender countries were Spain, Greece, Turkey, Italy, Portugal and ex-Yugoslavia. In November 1973, the program was formally closed and immigration to Germany continued mainly via other channels such as family reunification. Despite the temporary nature of the guest-worker program, many immigrants did not return to their home countries, since economic conditions were not favorable there. In recent years, Germany continued to attract a large number of immigrants. In the past few years, the number and composition of immigrants in Germany has been affected by events such as the EU enlargement and the large waves of refugees and asylum seekers.

According to the OECD, the inflow of immigrants in Germany was above one million in 2013, albeit among them only about 450,000 were estimated to be "permanent immigrants" (OECD, 2015). ${ }^{10}$ Immigrants originate both from EU and non-EU countries. Due to historical factors that determined the initial location of immigrants and also as a consequence of their subsequent internal migration, the immigrant population is not evenly distributed in Germany. Moreover, immigrants are composed by a large number of different nationalities, with a level of ethnic diversity that varies substantially across regions.

Figure 1 provides some initial insight about ethnic diversity and its evolution over time. The graph represents the ED index, whereby values are weighted by the population size in

\footnotetext{
${ }^{10}$ Estimates from the OECD were taken from https://stats.oecd.org/Index.aspx?DataSetCode=MIG. Last access September $4^{\text {th }} 2016$.
} 
each ROR and year to make the index representative for the whole Germany. Ethnic diversity is already high at the beginning of the period of interest (above 0.87), and it further increases over fifteen years to reach a value above 0.92. There are various factors behind the increase of the index, including a rise in immigration levels, the number of nationalities present in Germany and the redistribution of foreign nationals across regions of Germany.

Figure 1: Ethnic diversity, 1998-2012

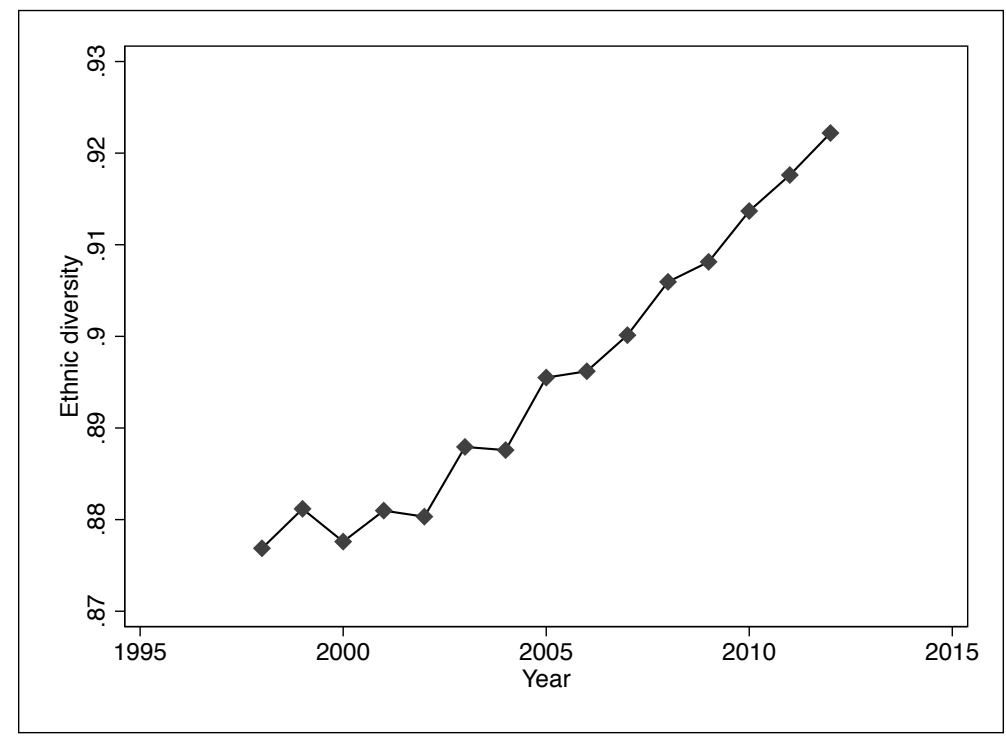

Notes: Data refer to the ED index for Germany over 1998 to 2012, weighted by the size of population in each ROR/year.

In Figure 2 we show the spatial distribution of the ED index along with other regional indicators. The top two panels show maps of the ED index in 1998 and 2012, the first and last year of our analysis. Darker areas represent higher values. Some areas exhibit relatively low ethnic diversity in both periods (e.g., a few RORs in the North Rhine-Westphalia and Baden-Wurttemberg states). At the same time, there are RORs with high level of ethnic diversity both in 1998 and in 2012 (e.g., most RORs in Saxony). Finally, other RORs experienced either a decrease (e.g., several RORs in Lower Saxony) or an increase (e.g., in Thuringia) of the ED index.

The bottom panel shows the immigrant share, i.e., the number of immigrants over the total resident population in each ROR, and the male unemployment rate. Data refer to averages over the period 1998-2012. Immigration (rather contrary to ethnic diversity) is more pronounced in West than East Germany. There is also substantial variation within states, but only in West Germany. At the same time, unemployment rates are far higher in the East, while they are lower in the West, but with marked differences across and within States. These maps suggest that the relationship between ethnic diversity and other regional indicators is 
Figure 2: ROR characteristics

Ethnic diversity, 1998

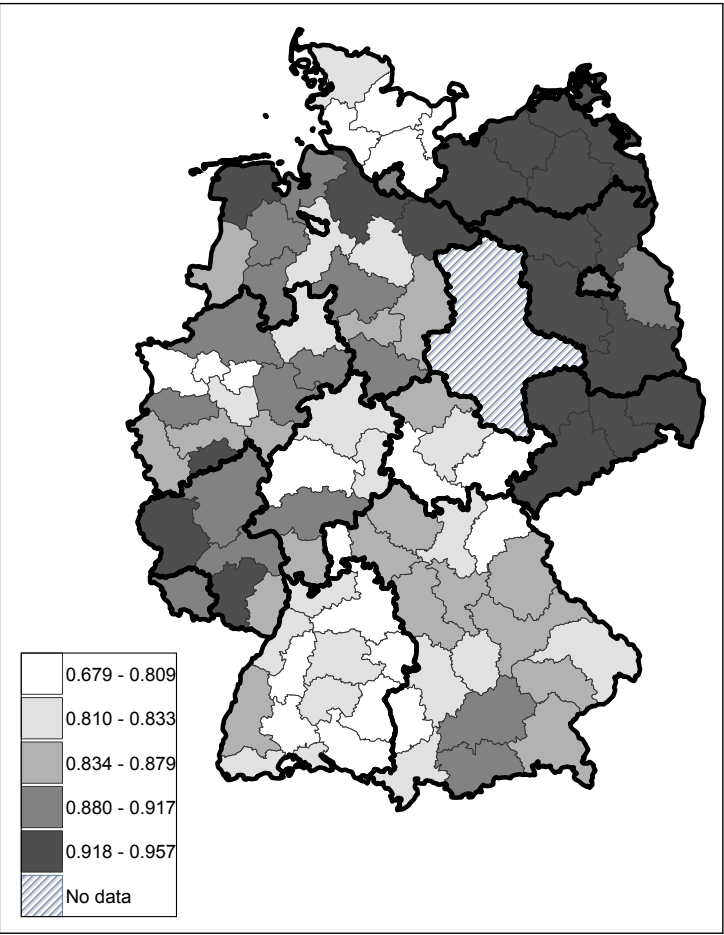

Immigrant share

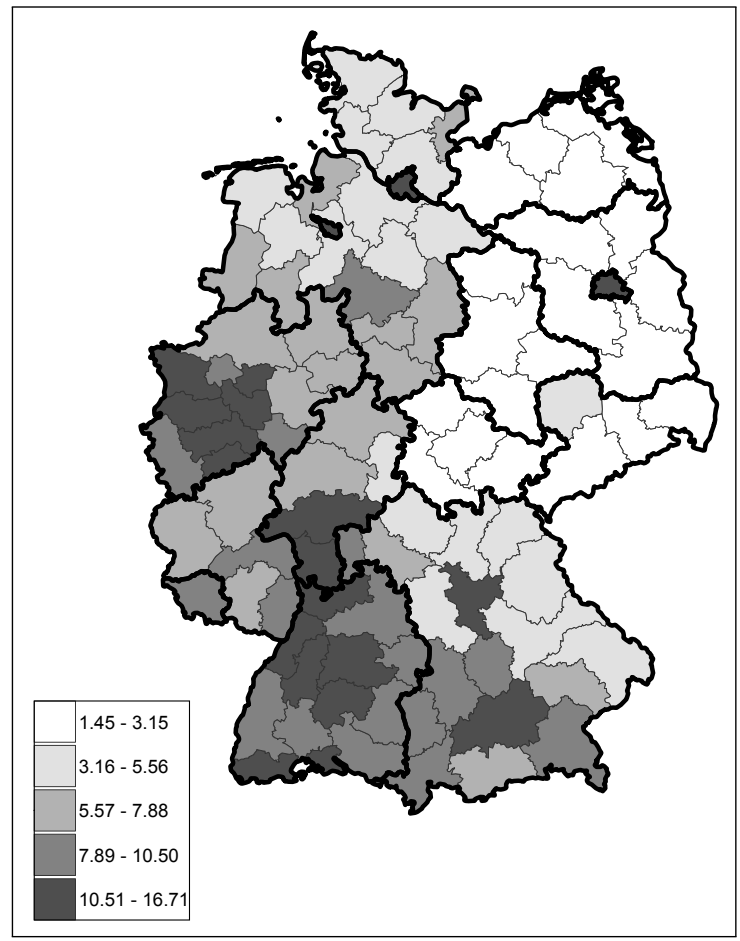

Ethnic diversity, 2012

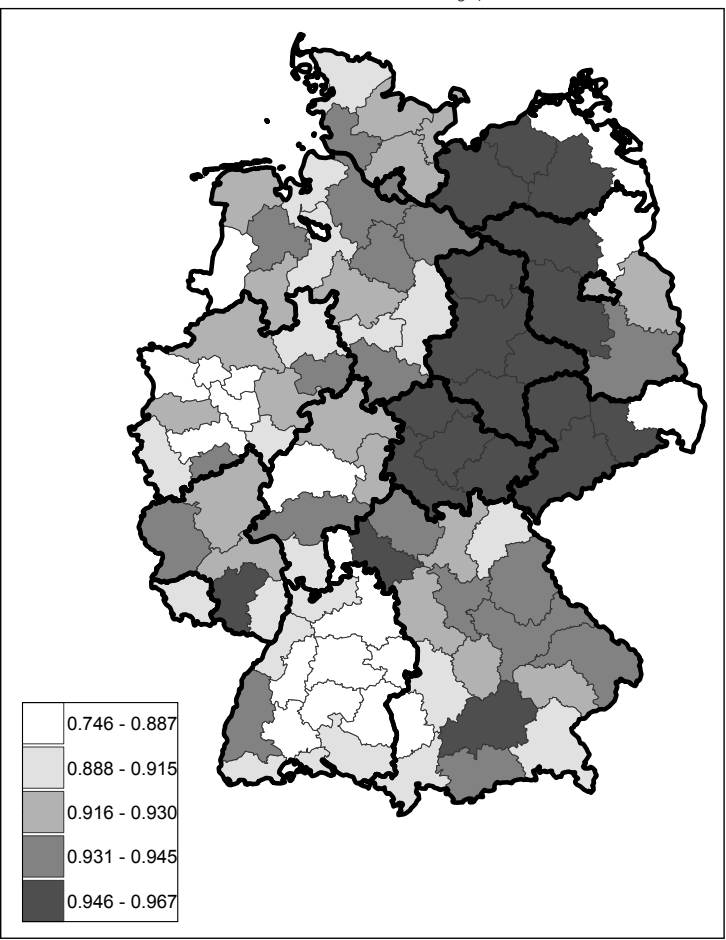

Unemployment rate

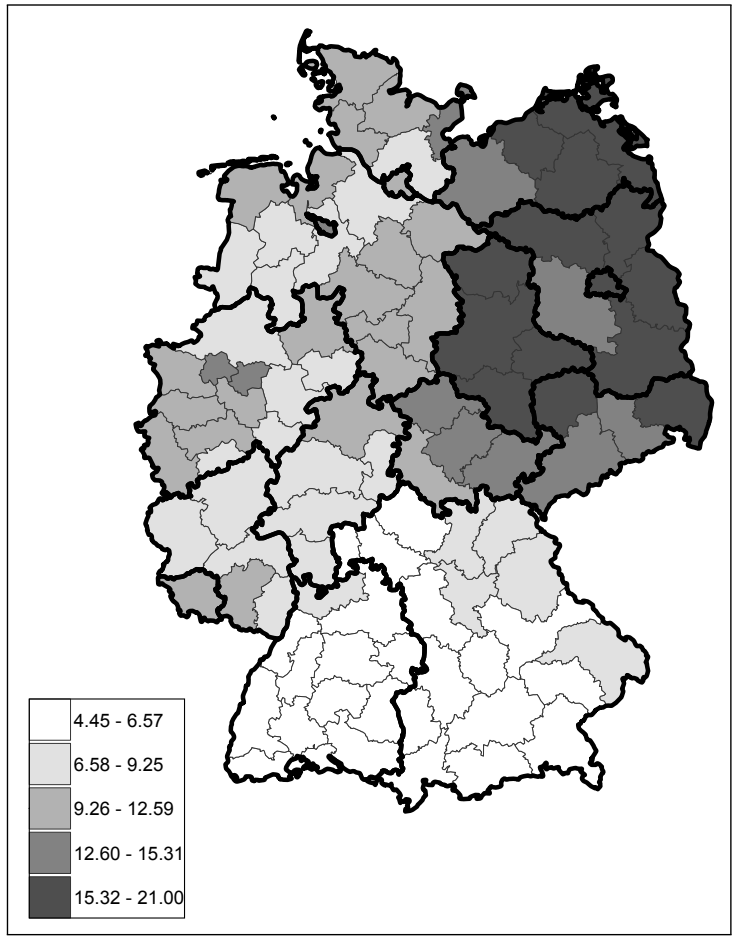

Notes: Data for Sachsen-Anhalt are available only from 2007. 
somewhat complex, since in some areas (e.g., East Germany) diversity is relatively more pronounced where immigration is more intense and unemployment is high, while in West Germany this pattern is less obvious.

\subsection{Key Characteristics}

Table 1 presents the descriptive statistics of our sample. We report averages and standard deviations of SWB and ethnic diversity, as well as individual and regional characteristics for the whole sample, the first and final year of analysis. The asterisks in the last column indicate whether the 1998 and 2012 averages are statistically different from each other at the 0.01 significance level. The overall level of SWB is about 7, in line with previous studies using the same dataset (e.g., Ferrer-i Carbonell, 2005, Akay et al., 2014). We also observe changes in reported well-being over time. The well-being level is 6.98 in 1998 while it is 7.08 in 2012, with a statistically significant difference. The remaining individual characteristics are also similar to those used in other well-being studies based on the GSOEP. However, there are interesting changes over time. For example, the share of natives without children increased from 0.58 to 0.68 during the period of interest, while the percentage of married individuals decreased by about 5 percentage points. The share of employed natives increased from 0.69 to 0.76 , and wages increased by about 10 percent. These changes partly reflect the "ageing" of the sample (e.g., the average age in 2012 is 5 years higher than in 1998). In addition, during the period of analysis there were many changes in the economic conditions of the country, including labor market reforms that affected outcomes such as employment and wages. In the lower part of Table 1, we show averages of the regional characteristics. Ethnic diversity increased over the years, as already observed in Figure 1. The immigrant share is about $8.21 \%$, with a value slightly higher in the initial period. The overall male unemployment rate in the region is about 11\%, but with substantially lower levels in 2012 than in 1998. The foreigners' unemployment rate is, on average, lower than the male unemployment rate. However, it follows a different path over time, as it is higher in 2012 than in 1998.

\section{Econometric Specifications}

The dependent variable used in our analysis is the individuals' subjective well-being, which is a latent variable, yet is observed with an ordinal metric. The baseline regression model is:

$$
\begin{aligned}
S W B_{i t}^{*} & =\beta E D_{r t}+\phi I M_{r t}+\mathbf{Z}_{r t}^{\prime} \boldsymbol{\lambda}+\mathbf{X}_{i t}^{\prime} \boldsymbol{\gamma}+\varepsilon_{i t} \\
\varepsilon_{i t} & =\rho_{r}+\tau_{t}+\alpha_{i}+\nu_{i t}
\end{aligned}
$$


Table 1: Summary statistics

\begin{tabular}{|c|c|c|c|c|c|c|c|}
\hline \multirow[b]{2}{*}{ SWB } & \multicolumn{2}{|c|}{ All } & \multicolumn{2}{|c|}{1998} & \multicolumn{2}{|c|}{2012} & \multirow[b]{2}{*}{$*$} \\
\hline & 7.0163 & $(1.7357)$ & 6.9818 & $(1.7165)$ & 7.0864 & $(1.6846)$ & \\
\hline \multicolumn{8}{|l|}{ Individual characteristics } \\
\hline Age & 42.158 & $(12.79)$ & 39.572 & $(12.931)$ & 44.185 & $(12.84)$ & $*$ \\
\hline Females (\%) & 0.5157 & $(0.4998)$ & 0.5081 & $(0.5)$ & 0.5186 & $(0.4997)$ & \\
\hline East Germany (\%) & 0.2529 & $(0.4347)$ & 0.2881 & $(0.4529)$ & 0.2634 & $(0.4405)$ & $*$ \\
\hline Years of education/training & 12.400 & $(2.632)$ & 11.811 & $(2.452)$ & 12.723 & $(2.71)$ & * \\
\hline Household size & 2.8637 & $(1.2427)$ & 2.9632 & $(1.2212)$ & 2.6950 & $(1.2284)$ & $*$ \\
\hline No children (\%) & 0.6245 & $(0.4842)$ & 0.5791 & $(0.4937)$ & 0.6759 & $(0.4681)$ & $*$ \\
\hline One child (\%) & 0.1944 & $(0.3958)$ & 0.2224 & $(0.4159)$ & 0.1731 & $(0.3783)$ & * \\
\hline Two children (\%) & 0.1401 & $(0.3471)$ & 0.1513 & $(0.3584)$ & 0.1195 & $(0.3244)$ & $*$ \\
\hline Three or more children (\%) & 0.0409 & $(0.1982)$ & 0.0472 & $(0.212)$ & 0.0315 & $(0.1747)$ & $*$ \\
\hline Married (\%) & 0.5947 & $(0.4909)$ & 0.6090 & $(0.488)$ & 0.5515 & $(0.4974)$ & $*$ \\
\hline Separated (\%) & 0.0208 & $(0.1426)$ & 0.0184 & $(0.1346)$ & 0.0244 & $(0.1542)$ & * \\
\hline Single $(\%)$ & 0.2874 & $(0.4525)$ & 0.2894 & $(0.4535)$ & 0.3037 & $(0.4599)$ & \\
\hline Divorced (\%) & 0.0779 & $(0.268)$ & 0.0628 & $(0.2426)$ & 0.0980 & $(0.2974)$ & $*$ \\
\hline Widowed (\%) & 0.0193 & $(0.1375)$ & 0.0203 & $(0.1412)$ & 0.0224 & $(0.1479)$ & \\
\hline Very good health (\%) & 0.1068 & $(0.3088)$ & 0.1132 & $(0.3169)$ & 0.0994 & $(0.2992)$ & * \\
\hline Good health (\%) & 0.4564 & $(0.4981)$ & 0.4743 & $(0.4994)$ & 0.4463 & $(0.4971)$ & $*$ \\
\hline Satisfactory health (\%) & 0.3083 & $(0.4618)$ & 0.2934 & $(0.4553)$ & 0.3185 & $(0.4659)$ & $*$ \\
\hline Poor health (\%) & 0.1059 & $(0.1059)$ & 0.0979 & $(0.0979)$ & 0.1086 & $(0.1086)$ & $*$ \\
\hline Bad health (\%) & 0.0227 & $(0.1488)$ & 0.0211 & $(0.1438)$ & 0.0271 & $(0.1623)$ & $*$ \\
\hline Employed (\%) & 0.7261 & $(0.446)$ & 0.6865 & $(0.4639)$ & 0.7580 & $(0.4283)$ & $*$ \\
\hline Not in labour force (\%) & 0.1855 & $(0.3887)$ & 0.2068 & $(0.405)$ & 0.1581 & $(0.3648)$ & * \\
\hline In school or training (\%) & 0.0318 & $(0.1753)$ & 0.0358 & $(0.1857)$ & 0.0325 & $(0.1773)$ & \\
\hline Unemployed (\%) & 0.0567 & $(0.2313)$ & 0.0710 & $(0.2568)$ & 0.0514 & $(0.2209)$ & $*$ \\
\hline Wages (log) & 7.9277 & $(3.9961)$ & 7.4893 & $(4.1187)$ & 8.2949 & $(3.8054)$ & $*$ \\
\hline Hours worked (log) & 2.5279 & $(1.6726)$ & 2.3716 & $(1.7533)$ & 2.6517 & $(1.6039)$ & $*$ \\
\hline Household income (log) & 7.5425 & $(1.9175)$ & 7.8781 & $(1.8718)$ & 7.5208 & $(1.7933)$ & $*$ \\
\hline \multicolumn{8}{|l|}{ Regional variables } \\
\hline Ethnic diversity & 0.8884 & $(0.0533)$ & 0.8658 & $(0.0583)$ & 0.9181 & $(0.0381)$ & $*$ \\
\hline Immigrant share & 8.2099 & $(4.6016)$ & 8.1403 & $(4.9384)$ & 7.5312 & $(4.4991)$ & $*$ \\
\hline Unemployment rate & 10.7812 & $(4.8846)$ & 12.3580 & $(3.8643)$ & 6.6728 & $(3.1841)$ & * \\
\hline Immigrant unemployment rate & 9.2411 & $(2.7606)$ & 8.8869 & $(2.4479)$ & 9.1112 & $(3.0242)$ & $*$ \\
\hline Log GDP & 3.2535 & $(0.2787)$ & 3.0879 & $(0.2788)$ & 3.4383 & $(0.2393)$ & $*$ \\
\hline $\mathrm{N}$ & \multicolumn{2}{|c|}{188,123} & \multicolumn{2}{|c|}{8,947} & \multicolumn{2}{|c|}{11,487} & \\
\hline
\end{tabular}

Source: GSOEP 1998-2012. * indicates whether the 1998 and 2012 averages are statistically different from each other at the 0.01 significance level.

where $i$ indicates the individual and $t$ the year. Ethnic diversity $(E D)$ is measured for each $\operatorname{ROR}(r)$ and year. $\beta$ is the key parameter of our analysis. To identify the relationship between ethnic diversity and well-being, we control for several characteristics. The baseline specification includes the immigrant share $(I M)$ and several regional attributes $(\mathbf{Z})$, such as GDP per capita and unemployment rates. The matrix $\mathbf{X}$ contains a rich set of individual and household covariates, (see Table 1 for the full set of control variables).

The error term $\varepsilon$ includes several components. First, it includes the ROR fixed-effects (96 regional dummies, indicated by $\rho$ ) in order to control for regional unobserved confounders. Second, to be able to account for period-specific changes in the overall economy or in political 
conditions, we add year dummies $(\tau)$. Third, we allow for individual unobserved heterogeneity $(\alpha)$, which is assumed to be correlated with ethnic diversity. In general, unobserved individual characteristics can substantially influence SWB (Boyce et al., 2010). In our settings, it is particularly important to control for unobservable heterogeneity since there could be various selection mechanisms due to omitted variables correlated with changes in ED over time.

While our preferred specification uses individual fixed-effects, we consider some alternative ones. We compare our results with those of an ordered probit model (OP). Differences between an ordered probit and a linear specification can be ignored if there are relatively large number of categories (see e.g., Ferrer-i Carbonell and Frijters, 2004). The advantage of linear regression is the possibility of using the panel dimension of the data and include unobserved individual heterogeneity in a more flexible way (e.g., Diener et al., 1999, Akay and Martinsson, 2012, 2009). We then check the results vis-à-vis those from alternative models in which unobserved heterogeneity is also accounted for. We estimate a standard random-effects model (RE) and one in which we specify a flexible auxiliary distribution for the unobserved individual effects following the correlated random-effects model (also known as quasi-fixed-effects - QFE). This specification allows flexibility on the relationship between time-variant characteristics and unobserved individual effects. ${ }^{11}$ Finally, we compare the results with those from standard OLS.

\section{Results}

In this section, we present the results of our analysis. First, we show our baseline estimates, including the preferred fixed-effects specification. We then outline the results from regressions using alternative definitions of ethnic diversity. Subsequently, we investigate whether internal mobility constitutes a potential threat to a causal interpretation of our results. Finally, we explore the heterogeneity of results across different socio-demographic groups and personality traits. We discuss our results and potential mechanisms in the next Section.

A Quick Look at the Determinants of SWB. Throughout the analysis, we present only the estimates of the key parameters. Table A1 in the Appendix reports the estimates of all covariates used in the regressions. We now briefly describe the estimates of a few characteristics which have been explored in previous SWB studies.

Socio-demographic and economic determinants of SWB are in line with the results reported in studies that use similar specifications and data (e.g., Frey and Stutzer, 2002,

\footnotetext{
${ }^{11}$ The time-variant characteristics that we use for the QFE specification are averages over time of household size, household income after tax and weekly working hours.
} 
Ferrer-i Carbonell, 2005, Dolan et al., 2008, Akay and Martinsson, 2012). Having good health, more years of education, being married and employed and possessing a relatively high income are factors that have a positive relationship with SWB. Residents in East Germany report lower levels of SWB (a pattern already seen in Frijters et al., 2004). Our data confirm the existence of the well-known U-shape relationship between age and SWB (e.g., Blanchflower and Oswald, 2008, Graham and Pozuelo, 2017), with the "minimum" level of happiness occurring around the age 40-45.

\subsection{Ethnic Diversity and Subjective Well-Being}

Baseline Estimates. We now present the estimates of our regression analysis. The baseline results are shown in Table 2. All specifications contain the individual-level characteristics reported in Table 1, as well as indicators for RORs and years. The first three columns are estimated with individual fixed-effects. With the exception of the model in the first column - in which we only include the immigrant share - we also control for ROR-level time-varying attributes. We cluster the standard errors at the ROR-year level, given that these are the dimensions at which ethnic diversity is measured. Our preferred specification is the one in the second column, namely a fixed-effect model with all ROR controls.

The results of the fixed-effects model show that the parameter estimates of ethnic diversity are positive and significant at the $1 \%$ significance level. We will discuss the size of the effect in the next subsection. For now it is interesting to note that fixed-effects estimates with and without regional controls are only marginally different. The positive estimates suggest that ethnic diversity is associated with welfare gains for Germans. This result complements the finding of Akay et al. (2014), who discovered a positive effect of the immigrant share in the region on the well-being of natives. We confirm the existence of such positive relationship also in our sample, which contains more years, and even after introducing ethnic diversity in the regression. In column III we estimate a model where we include an interaction term between ethnic diversity and immigrant share. ${ }^{12}$ The estimates show that the interaction is negative - although moderate. The interpretation that we privilege is that migration size and composition are substitutes. One way of thinking about this result is that natives' well-being increases through either a large number or a large variety of immigrants. In other words, in areas where immigrants are relatively underrepresented, having a large variety of immigrant nationalities "compensates" for the size effect.

To better understand the relevance of the interaction between ethnic diversity and immigrant share, in Table A2 in the Appendix we report predicted values of well-being (and

\footnotetext{
${ }^{12}$ From the maps in Figure 2, one notices that ethnic diversity and immigrant share are somewhat negatively correlated. The estimated correlation is -0.25 .
} 
related prediction confidence intervals) for both the model without and with interaction effects (i.e., the specifications in columns II and III of Table 2, respectively). To obtain these, we first split the ED index and the immigrant share into four quartiles, and use the mean point of each quartile to predict the well-being (hence generating 16 possible combinations for the two variables). When looking at the interaction model (reported in the second panel of Table A2, one notices that predicted values are relatively larger when either the immigrant share or the ethnic diversity is larger. This is not the case with the main effects model, where predicted values monotonically increase with both the ethnic diversity and the immigrant share. Despite the dissimilar pattern, however, fitted values are not substantially different between the two models.

Overall, the estimates in the first three columns of Table 2 suggest that both the size (immigrant share) and the composition (ethnic diversity) of immigration matter for the well-being of natives.

Estimators. Our baseline fixed-effects (FE) specification allows controlling for several confounders potentially correlated with ethnic diversity. In order to compare the sensitivity of our preferred estimates to alternative estimators, we provide additional results in the remaining columns of Table 2. First, we estimate an ordered probit model (OP) without allowing for unobserved individual heterogeneity. We remind the reader that parameter estimates of an ordered probit and of a linear model cannot be directly contrasted. Nevertheless, the comparison of signs and statistical significance is insightful to understand how a different estimator would affect our results. As can be noted from the estimates in column IV, the sign and significance of the results are similar to those in the first three columns. ${ }^{13}$ The next two columns present the results using random-effects estimators. We consider both standard random-effect (RE) and quasi-fixed-effects (QFE) models. The estimates from these two models do not substantially differ from those of our preferred specification. ${ }^{14}$ Lastly, we show results from the linear model estimated with OLS. Even in this case, the estimates of ethnic diversity are not too dissimilar from the preferred specification.

\footnotetext{
${ }^{13}$ We have also estimated a "Blow and Cluster" fixed-effects ordered logit model (Baetschmann et al., 2015). The aim of this specification is to allow controlling for individual heterogeneity by taking the ordinal nature of SWB into account. Note that in this model - very much like in the fixed-effects model - we omit time-invariant characteristics such as sex and those that are (quasi-)collinear with the time dummies, such as age. The estimate is 1.3319 (s.e. 0.3163), which suggests once again a positive and statistically significant relationship between ethnic diversity and well-being. Even in this case the point estimate is only qualitatively comparable with those of the fixed-effects model.

${ }^{14}$ We have performed an Hausman test between the FE and RE models and between the FE and QFE models, finding that in both cases we cannot reject the hypothesis that the FE model provides consistent estimates.
} 
Table 2: Multiculturality and happiness - regression results

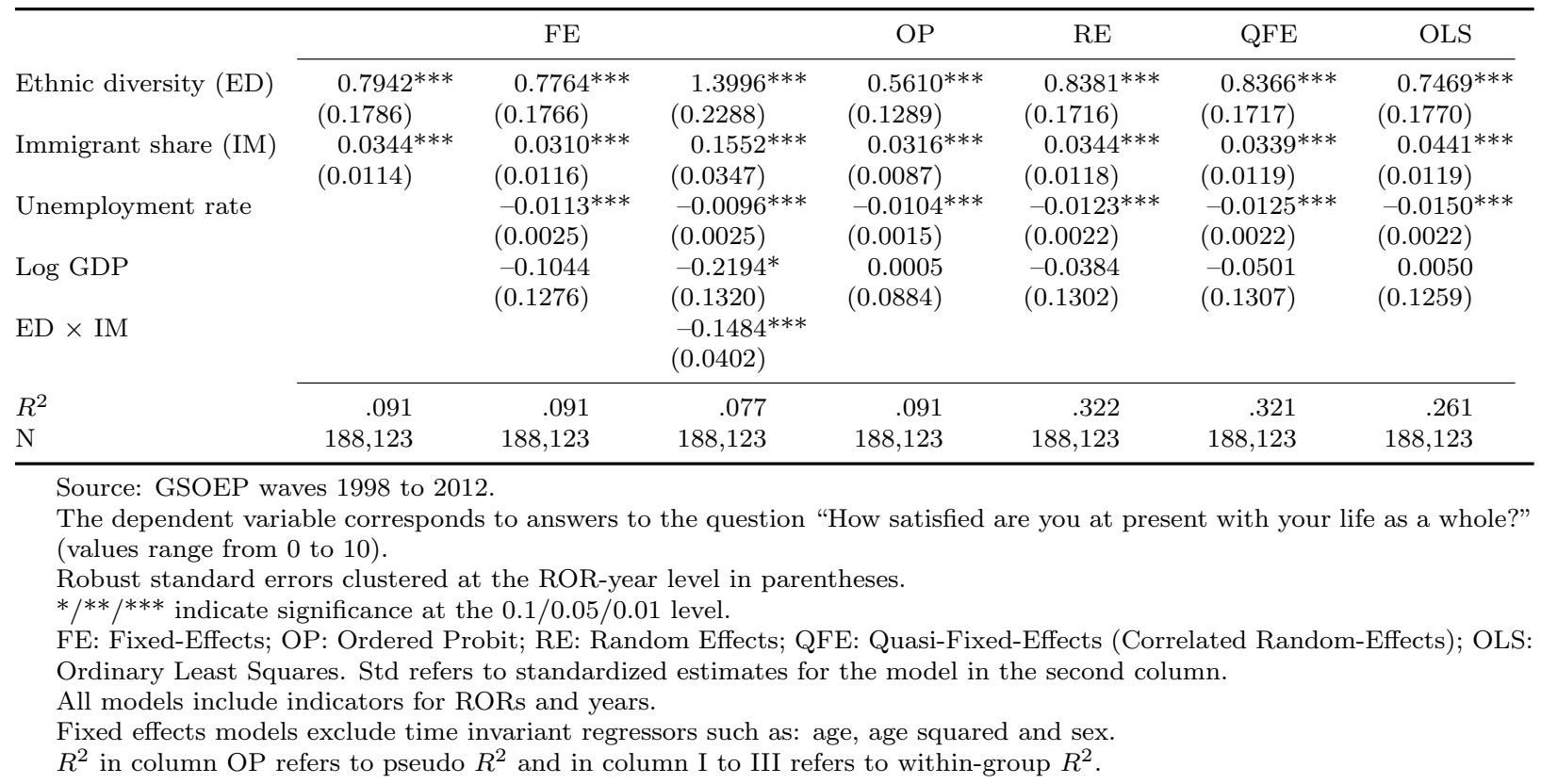

Is the Effect Large? To provide an idea about the magnitude of the effect, we calculate the standardized coefficients for our preferred specification and report them in the last column of Table A1 in the Appendix. The estimates indicate that one standard deviation change in ED is associated with 0.023 standard deviation change in SWB. This value can be better explained by comparing it with other covariates. For example, the magnitude of ethnic diversity is similar or even larger than that of other SWB determinants such as household income (0.013) and working hours (0.029). However, it is relatively smaller when compared to other important factors such as being unemployed (-0.070) and the immigrant share (0.082).

\subsection{Alternative Definitions of Ethnic Diversity and Sensitivity Checks.}

Thus far we have used a measure of ethnic diversity based on the Herfindahl-Hirschman index. However, the literature has explored also other measures (see e.g., Massey and Denton, 1988, McDonald and Dimmick, 2003). Furthermore, one might wonder how the effect would change depending on the number or the grouping of nationalities. In this subsection, we explore alternative measures and definitions of ethnic diversity.

Shannon Entropy Index and Segregation. To check the sensitivity of the results, we estimated our preferred specification using the Shannon Entropy (SE) index - another widely-used diversity measure (e.g., Lande, 1996, McCulloch, 2007). This is defined as: 


$$
S E_{r}=-\sum_{g} \frac{m_{g r}}{m_{r}} \ln \left(\frac{m_{g r}}{m_{r}}\right)
$$

where $m_{g r}$ and $m_{r}$ are defined as for the ED index. A higher value of $S E_{r}$ implies a higher level of ethnic diversity. It can be easily shown that the maximum level of the index corresponds to the log of the number of ethnic groups $g$.

In Panel A of Table 3 we present the results of our preferred specification using the Shannon Entropy index. The first column shows a positive and statistically significant estimate. Perhaps this is not so surprising, given that the correlation between the SE and ED indices is 0.89. An important implicit assumption underlying both diversity measures is that the spatial distribution of the ethnic groups within the same regions is homogenous. This assumption might not hold if, say, immigrants would segregate into particular areas within a region. An important property of the SE index is that it can be decomposed into a part that captures the diversity within each region (within-area diversity) and a part that measures the diversity between sub-regions, which can tell us how ethnic groups spatially segregate (between-area diversity). Following Lande (1996) and McCulloch (2007), the SE index can be decomposed as:

$$
S E_{r}=-\sum_{g} \pi_{g r} \ln \left(\pi_{g r}\right)=-\sum_{k} \pi_{k r}\left[\sum_{g} \pi_{g k} \ln \left(\pi_{g k}\right)\right]+\sum_{k} \pi_{k r}\left[\sum_{g} \pi_{g k} \ln \left(\frac{\pi_{g k}}{\pi_{g r}}\right)\right]
$$

where $\pi_{k r}=m_{k r} / m_{r}, \pi_{g k}=m_{g k} / m_{k}$, and $\pi_{g r}=m_{g r} / m_{r}$. Here $k$ represents sub-regions. In practice, the Shannon Entropy index corresponds to the linear combination of the within and between component, weighted by the relative shares of immigrants in the area. The first part of the decomposition captures the degree of the mix of ethnic groups in "absence" of segregation, i.e., if the share of an ethnic group in each sub-region $\left(\pi_{g k}\right)$ is the same of the share of the same group in the region $\left(\pi_{g r}\right)$. The second part reflects segregation, i.e., the scenario that each sub-area was composed by one ethnic group only. ${ }^{15}$

We derive the two components of the SE index by exploiting nationality data at the district level. This allows us obtaining the two components for all 96 RORs over time and use them to repeat our baseline analysis. As Figure 3 shows, the within-area component - very much like the overall SE and ED indices - has been increasing over time. On the contrary, the between-area component has remained rather stable over the years.

\footnotetext{
${ }^{15}$ Glitz (2014) reports that both workplace and residential segregation among natives and immigrants are persistent over time, with the former being more pronounced. The author also shows that residential segregation does not vary by skills of immigrants, but differences are observed across nationalities, with Turkish, Greek and African immigrants being the groups that are more segregated.
} 
Figure 3: Shannon Entropy index and components

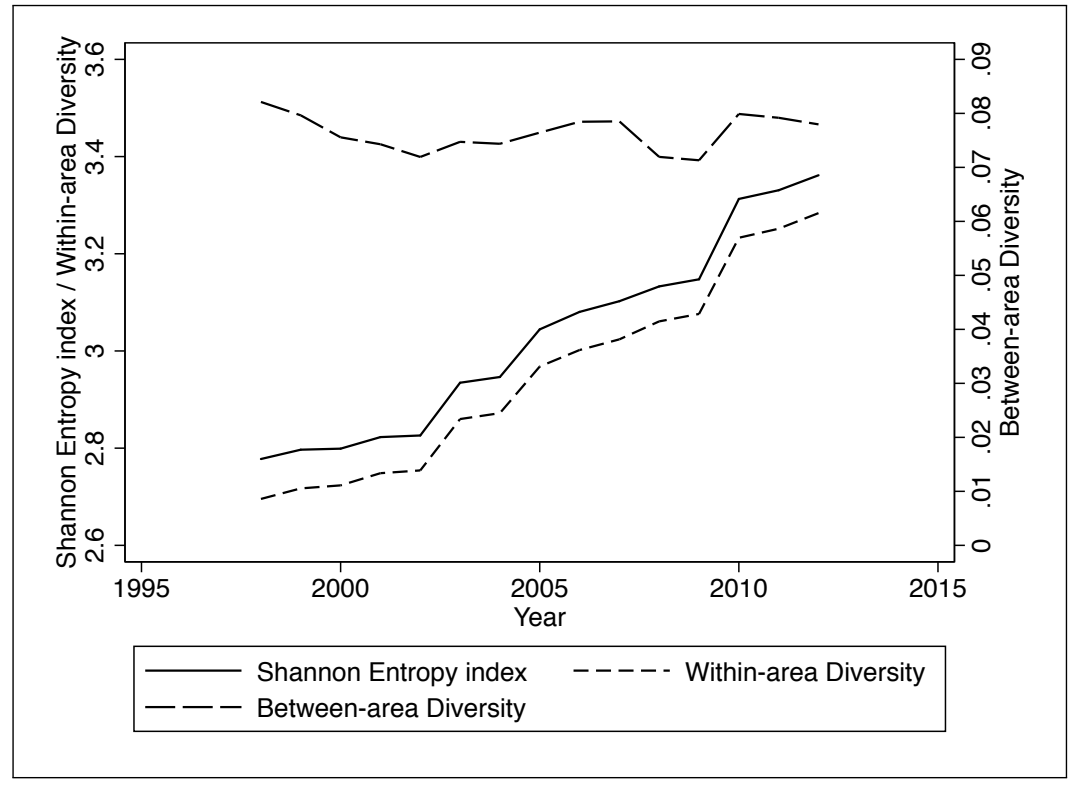

Notes: Data refer to the average Shannon Entropy index for Germany over 1998 to 2012 .

The remaining columns of Panel A in Table 3 contain the estimates of regressions where we use the within-area component, the between-area component and both of them in separate model specifications. The estimate of the within-area component is positive and significant. Perhaps this is not so surprising, given that this measure is highly correlated with the Shannon index. However, Germans' well-being is found to be negatively associated with the between-area component. These results are confirmed when both components are used in the same regression. This suggests that, while Germans are happy with ethnic diversity overall, segregation is associated with a loss of welfare. The negative impact of segregation is, on average, small and not large enough to compensate the positive impact of within-area diversity. Yet, the welfare loss can be relatively large in areas where immigrants tend to be particularly isolated. The map in Figure A1 in the Appendix indicates that such areas are scattered both in East and West Germany. This is in contrast to the spatial distribution of the within-area component, which resembles that of the ED index in Figure 1. Note that the correlation between the two components of the Shannon index is below 0.12.

Cultural and economic distance. Panel B of Table 3 explores the potential role of cultural/economic proximity. One underlining assumption of our ED index is that all nationalities are of equal "distance" in terms of their culture from the host population. This might not be the case for Germany and many other high immigration countries that receive flows of immigrants from nearly all countries in the world. Similarly, among the nationalities 
used in the calculations of the ED index there are those that are as or more economically developed than Germany and those that are less rich. To account for this heterogeneity, we "weigh" the ED index with measures of cultural and economic proximity of immigrants.

We follow a procedure similar to that of Alesina et al., 2016 who construct a cultural proximity measure using the genetic distance data collected by Spolaore and Wacziarg (2009) and an economic proximity measure using per-capita GDP data. First, we obtained the most recent genetic distance data (see Spolaore and Wacziarg, 2016). From the database, we selected only the values of the genetic distance between Germany and the (available) remaining countries in the world. We then normalize the values and bound them between 0 and 1. This index of genetic distance can be thought of as a proxy for the biological and cultural differences between Germany and the other countries. We use this index to derive the weight $\omega_{g}=2 /\left(1+e^{-d_{g} \times q}\right)$. Here $d_{g}$ represents the normalized genetic distance of each nationality $g$ from Germany and $q$ is a parameter that can take several values. Hence $\omega$ is bounded between 0 and 2. For our purposes, we use $q=-10$ and $q=10$, the two "extreme" values adopted in Alesina et al. (2016). Finally, we use the weight $\omega_{g}$ to multiply the components of the ED index. ${ }^{16}$ The rationale of this weighting is to give more or less importance to nationalities of a certain genetic/cultural distance from Germany.

After constructing an ED index weighted by cultural proximity, we obtained a similar one based on economic distance, making use of per-capita GDP data for $2013 .{ }^{17}$ We construct a relative measure of GDP by taking the ratio between each country GDP and that of Germany. We normalize this measure in order to obtain an index which varies between 0 and 1 . We then proceed to create the weight $\omega_{g}$ similarly to what done above.

In panel B of Table 3, we report the results of our analysis using the ED index weighted for cultural and economic proximity. Column I presents the scenario of giving more importance to nationalities which are culturally closer (i.e., $q=-10$ ), while column II refers to the opposite scenario. The estimates suggest that the effect of ethnic diversity is stronger for groups that are culturally closer to Germany. The results by income reveal stronger effects when more weight is given to relative richer countries (column III), while the estimate is economically and statistically weaker when more importance is given to poorer countries (column IV). These results are consistent with those of Alesina et al. (2016), who also found that the effect of birthplace diversity on economic growth is stronger when more weight is given to groups who are economically and culturally closer.

\footnotetext{
${ }^{16}$ The weighted ED index can be written as $E D_{r}=\sum_{g}\left(\frac{m_{g r}}{m_{r}}\right) \times\left(1-\frac{m_{g r}}{m_{r}}\right) \times \omega_{g}$

${ }^{17}$ Data were obtained from http://wdi.worldbank.org.
} 
Alternative nationality groupings. In Panel $\mathrm{C}$ of Table 3 we provide further tests to explore the sensitivity of our results to alternative groupings of nationalities. First, one might think that the results are sensitive to the number of nationalities included, and possibly driven by small immigrant groups. This check is particularly relevant since the number of nationalities used in our dataset varies across regions and years. Furthermore, the number of nationalities was less uniform before data collection was harmonized at the Federal level in 2007. Second, one might think that certain immigrant groups are more alike than others (in terms of e.g., culture, economic development, language, religion and race) and hence diversity should be measured by aggregating nationalities which shares the same characteristics.

In column I we use only the top ten nationalities of immigrants in Germany. This means that the diversity index is calculated using these ten nationalities and an eleventh residual category. ${ }^{18}$ The advantage of using such measure is that the ED index is homogenous (i.e., defined using the same number of groups) across all RORs and over all years. The resulting estimates are qualitatively similar to the preferred specification in column II of Table 2.

In column II and III we estimate models where the ED index is defined using broad regions. In column II we use the following eight regions: EU-15, other Western countries, Eastern Europe, Turkey, Arab countries, remaining Africa, Latin America, Asia and rest of the world. These can be thought as areas with marked differences in terms of economic, racial, religious and cultural characteristics. In column III, we distinguish between EU and non-EU countries. The point estimates of these two tests are not dissimilar from the baseline.

Overall, the results of these three tests are interesting for two reasons. First, they show that measurement issues related to the number and grouping of available nationalities seems negligible. Second, diversity matters also when measured within broader regions of origin. This complements the evidence of Panel B since even within broader nationalities (e.g., EU and non-EU) there are groups that are culturally and economically closer to Germany (e.g., Italy and France for the EU and the US and Switzerland for the non-EU).

In column IV, we investigate whether the change in the source of nationality data - which were provided by the State statistical offices for the period up to and including 2007 and by the Federal statistical office after this period - influences our estimates. To this aim, we estimate a regression where the ED index is interacted with an indicator for whether the period of analysis is before 2008 or otherwise. The estimate for the period before 2008 (reported in column IV) is very similar to the baseline model. ${ }^{19}$

\footnotetext{
${ }^{18}$ According to the AZR data, the top ten nations in terms of immigrants in Germany are: Austria, France, Greece, Italy, Poland, Portugal, Serbia, Spain, Turkey and the UK.

${ }^{19}$ The estimate for the interaction term (which captures potential changes in the regime of data) is somewhat lower, but still positive and significant (0.613, s.e. 0.212).
} 
Table 3: Alternative measures of ethnic diversity and sensitivity checks

\begin{tabular}{|c|c|c|c|c|}
\hline \multirow[b]{3}{*}{ Shannon Entropy index } & \multicolumn{4}{|c|}{ Panel A: Shannon Entropy index and decomposition } \\
\hline & I & II & III & IV \\
\hline & $\begin{array}{l}0.0541^{* * *} \\
(0.0180)\end{array}$ & \multirow{3}{*}{$\begin{array}{l}0.0574^{* * *} \\
(0.0178)\end{array}$} & & \\
\hline Within diversity & & & & $\begin{array}{l}0.0582^{* * *} \\
(0.0174)\end{array}$ \\
\hline Between diversity & & & $\begin{array}{l}-0.5465^{* *} \\
(0.2519)\end{array}$ & $\begin{array}{l}-0.5618^{* *} \\
(0.2447)\end{array}$ \\
\hline$R^{2}$ & 0.091 & 0.091 & 0.091 & 0.091 \\
\hline \multirow[t]{3}{*}{$\mathrm{N}$} & 188,123 & 188,123 & 188,123 & 188,123 \\
\hline & \multicolumn{4}{|c|}{ Panel B: Genetic/cultural and economic distance } \\
\hline & I & II & III & IV \\
\hline Weighted thnic diversity & $\begin{array}{l}0.7389^{* * *} \\
(0.1896)\end{array}$ & $\begin{array}{l}0.5380^{* * *} \\
(0.1379)\end{array}$ & $\begin{array}{c}0.4895^{*} \\
(0.2652)\end{array}$ & $\begin{array}{l}0.6012^{* * *} \\
(0.1177)\end{array}$ \\
\hline$R^{2}$ & 0.091 & 0.091 & 0.091 & 0.091 \\
\hline \multirow[t]{3}{*}{$\mathrm{N}$} & 188,123 & 188,123 & 188,123 & 188,123 \\
\hline & \multicolumn{4}{|c|}{ Panel C: Sensitivity checks on ED definition } \\
\hline & I & II & III & IV \\
\hline Ethnic diversity & $\begin{array}{l}0.8331^{* * *} \\
(0.1819)\end{array}$ & $\begin{array}{l}0.8362^{* * *} \\
(0.1302)\end{array}$ & $\begin{array}{l}0.7982^{* * *} \\
(0.1377)\end{array}$ & $\begin{array}{l}0.7061^{* * *} \\
(0.1765)\end{array}$ \\
\hline$R^{2}$ & 0.091 & 0.091 & 0.091 & 0.091 \\
\hline \multirow[t]{3}{*}{$\mathrm{N}$} & 188,123 & 188,123 & 188,123 & 188,123 \\
\hline & \multicolumn{4}{|c|}{ Panel D: Sensitivity checks on regions } \\
\hline & I & II & III & IV \\
\hline Ethnic diversity & $\begin{array}{l}0.7953^{* * *} \\
(0.1771)\end{array}$ & $\begin{array}{l}0.8743^{* * *} \\
(0.1791)\end{array}$ & $\begin{array}{l}0.7516^{* *} \\
(0.2948)\end{array}$ & $\begin{array}{c}0.3862^{* *} \\
(0.1810)\end{array}$ \\
\hline$R^{2}$ & 0.091 & 0.092 & 0.096 & 0.081 \\
\hline $\mathrm{N}$ & 143,831 & 152,821 & 140,538 & 47,585 \\
\hline
\end{tabular}

Source: GSOEP waves 1998 to 2012.

The dependent variable corresponds to answers to the question "How satisfied are you at present with your life as a whole?" (values range from 0 to 10).

Robust standard errors clustered at the ROR-year level in parentheses.

$* / * * / * * *$ indicate significance at the $0.1 / 0.05 / 0.01$ level.

Panel A - Col I-IV: Ethnic diversity is measured using the Shannon Entropy index and its two components (see text for detailed explanation).

Panel B - Ethnic diversity is weighted using measures of genetic distance (I-II) and income (III-IV) of immigrants' nationalities (see text for detailed explanation).

Panel C - Col I: Ethnic diversity constructed using the ten nationalities present over time in all RORs (Austria, France, Greece, Italy, Poland, Portugal, Serbia, Spain, Turkey, UK); Col II: Ethnic diversity constructed using eight groups (EU15, other Western countries, Eastern Europe, Turkey, Arab countries, Africa (excluding Arab countries), Latin America, Asia and Other); Col III: Ethnic diversity constructed using only two broad nationalities (EU27 vs Other); Col IV: Interaction model with an indicator for the period when federal data are collected. The estimate for the interaction is .613 (s.e. .212)

Panel D - Col I: Sample excludes RORs that contain the top ten populated cities (Berlin, Hamburg, Munich, Cologne, Frankfurt, Stuttgart, Duesseldorf, Dortmund, Essen, Bremen); Col II: Sample excludes 5 RORs with highest average value of ethnic diversity (Trier, Havelland-Flaeming, Prignitz-Oberhavel, Oberes Elbtal/Osterzgebirge, West Saxony) and 5 RORs with highest average value of immigrant share (Hamburg, Rhine-Main, Stuttgart, Unterer Neckar and Munich); Col III: Residents in West Germany only; Col IV: Residents in East Germany only. All specifications are estimated with fixed-effects.

$R^{2}$ refers to within-group $R^{2}$. 
Further tests. In panel D of Table 3, we investigate the robustness of our results to the choice of regions. ${ }^{20}$ In column I we omit from the sample the RORs that include the ten largest cities of Germany. ${ }^{21}$ The reason for doing so is that big cities have large and ethnically diverse immigrant communities, and hence our results could be driven just by few areas. However, this does not seem to be the case, since the estimates are very similar to our baseline models. In column II we exclude the 5 RORs with the highest value of ethnic diversity and the 5 RORs with highest average value of immigrant share, finding again very similar results. ${ }^{22}$ Finally, in column III and IV we restrict our attention to either East or West Germany. The map in Figure 2 showed that diversity is higher in the East. At the same time, it shows that in the East there is much less spatial and time variation. This means that results could be completely driven by observations of residents in the West. The estimates of our last two tests show that while the effect is larger in West Germany, it is positive and statistically significant also in East Germany.

\subsection{Causality}

We now explore issues of natives' internal mobility that might confound our main finding and threaten its causal interpretation. It is important to remind the reader that our fixed-effects econometric strategy is already accounting for unobserved heterogeneity at the individual, regional and time dimensions. In the following, we consider two potential self-selection problems that could still affect causal interpretation.

The first is related to the possibility that high diversity in a region "attracts" or "pushes out" natives, leading them to internally migrate. This would be an issue if Germans' internal migration preferences were time-varying, since they would not be controlled by the individual fixed-effects. For example, it could be the case that at some point in time Germans were

\footnotetext{
${ }^{20}$ In unreported regressions, we test the sensitivity to the exclusion of second generation migrants from the sample of Germans. A subset of individuals, while reporting being born in Germany and having a German nationality, might feel attached to the country of origin of their parents. To explore the sensitivity of our results, we perform a check by excluding individuals born in Germany whose father or mother report being born abroad. Information on parental background is only available after 2006 and for a subset of individuals. Hence, we first perform a regression on the sample reporting parental background information (17,048 individuals), obtaining an estimate stronger than the baseline (1.240, s.e. 0.662). We then estimate a specification excluding second generation migrants (the sample decreases to 13,923 individuals), obtaining an estimate of 1.435 (s.e. 0.655). This result, although referred to a subset, suggests that including second generation migrants might annihilate the true effect of ethnic diversity.

${ }^{21}$ The top ten cities are: Berlin, Hamburg, Munich, Cologne, Frankfurt, Stuttgart, Dusseldorf, Dortmund, Essen, and Bremen.

${ }^{22}$ The 5 RORs with highest values of the ED index are: Trier, Havelland-Flaeming, Prignitz-Oberhavel, Oberes Elbtal/Osterzgebirge, West Saxony. The 5 RORs with highest values of immigrant share are: Hamburg, Rhine-Main, Stuttgart, Unterer Neckar and Munich. Excluding either the top 5 RORs in terms of ED index or in terms of immigrant share would produce similar results. Similarly, if we exclude the top 10 regions in terms of ethnic diversity and/or immigration, results would be very stable.
} 
unhappy with the high diversity in their region and decided to move to a region with less diversity. Then we would end up with a self-selected sample of Germans who would have higher than average happiness in ethnically diverse regions, and Germans with lower than average SWB in less diverse regions. If this selection was substantial, our positive estimate could be the byproduct of natives' internal migration. We would end up with an upward biased estimate if, for opposite reasons, Germans were attracted by more diverse regions.

The second issue concerns as to whether immigrants move within Germany depending on the SWB differences across regions. If certain groups of immigrants would move to happier regions, then the ethnic diversity would itself be a function of SWB, and this would lead to an overestimate of the positive effect. Akay et al. (2014) already showed that the internal mobility of immigrants is not affected by higher SWB in destination regions (and if anything, immigrants are pushed out from regions where more immigrants reside). This result notwithstanding, ethnic diversity could change in response to well-being differences even if migration does not. For example, if a certain number of Turks moves from region A to region $\mathrm{B}$, and the same number of Italians moves from region $\mathrm{B}$ to region $\mathrm{A}$, the overall immigrant share will not change. Yet, the level of ethnic diversity is likely to be different after the moves, depending on how many Italians and Turks there are in the two regions. To the extent that this redistribution is large, we might overstate the size of the true causal effect of ethnic diversity on happiness due to reverse causality bias. Our data allow us to explore whether these two issues are at work in our sample and the extent to which they affect our conclusions. We report results of the additional tests in Table 4.

Do Happy Natives Prefer Living in Ethnically Diverse Regions? In order to investigate whether this is the case, we estimate the probability that natives move within Germany as a function of the ethnic diversity differences between the ROR of destination (d) and that of origin (o), conditional on both observed and unobserved individual characteristics. To do so, we extract the subsample of Germans who have changed ROR at some point in time ( say $t$ ). For these individuals we can observe the characteristics of the ROR where they move to (d) and the one they come from (o). The characteristics of the latter are measured at time $t-1$. Since the same individuals are observed multiple times, we can still apply fixed-effects to allow for unobserved factors correlating with internal migration decisions. These settings yield a sample size of 18,097 individual $\times$ year observations. The specification is a linear probability model where the dependent variable takes the value of one if the natives moves and zero otherwise. The key explanatory variable is the difference between the ethnic diversity in the destination and in the origin. We also included the differences of other ROR variables. If Germans were attracted by the diversity in the ROR of 
destination (or unwilling to leave the ROR of origin) because of the high level of diversity, we would expect a positive correlation. This correlation would be negative in the opposite case, i.e., if Germans were "pushed" out from a region with high ethnic diversity (or attracted by a region with low ethnic diversity). The results in columns I-III of Table 4 show that while the point estimates are negative, they are far from being statistically significant. In the model with the full regional controls (column III), we note that regional differences in unemployment rates are actually the only variable that matters - with expected sign and large magnitude. Germans are less prone to move to a certain region if the unemployment rate is higher than in the origin region. Hence, we do not find evidence that our positive effect is the byproduct of the potential displacement effect of migration on natives.

Is Immigrants' Composition Affected by the Well-Being of Natives? We use a similar approach to investigate whether the internal mobility of immigrants is affected by regional differences in well-being. To this aim, we extracted from the GSOEP the sample of immigrants who report having changed RORs at least once over the period 1998 to 2012. First, we check whether immigrants respond to differences in the migration intensity and composition across regions. In columns IV-VI, we note that both the estimates of ethnic diversity and immigrant share are negative, but far from being significant, also considering the small sample size. One interpretation is that immigrants do not enjoy areas with high diversity. Instead, they like to move to areas where their own ethnic group represents the "majority" (e.g., ethnic enclaves). At the same time, they do not like to move to areas where there are many immigrants (of any group), perhaps due to stronger competition in the labor market (see e.g., Manacorda et al., 2012). This result confirms what Akay et al. (2014) found using a similar sample in Germany. The last row of these three columns includes the estimates for the regional difference in well-being. While estimates are positive across the three specifications, they are once again insignificant. Based on this, one could conclude that the internal mobility of immigrants (of all ethnic groups) does not substantially depend on whether the well-being in the destination region is higher than in the origin.

However, as pointed out above, even though differences in SWB might not affect the overall number of immigrants, they might still affect their composition, and thereby ethnic diversity. To explore this aspect more in depth, one would need to assess whether internal mobility patterns of each ethnic group are (or are not) affected by well-being. Ideally, we would like to split our sample across all available ethnic groups to investigate such question. However, the sample sizes would be too small. As a second best solution, in columns VIIIX, we perform a regression similar to that in columns IV-VII, and augment the model by introducing interaction terms between the largest ethnic groups and the variable represent- 
ing regional differences in well-being. These interactions can still be identified within our fixed-effects model since regional variables vary over time for each individual. The largest nationalities are: Turkey, Italy (reference group), Russia, Poland, ex-Yugoslavia, Kazakhstan and a residual group referred to as Other. ${ }^{23}$ The results show that only the interaction related to Turkey is statistically significant, but with a relatively large estimate. The sign of the estimate means that, with respect to the reference group, Turkish immigrants are more likely to move to regions with higher well-being (or out of regions with low well-being).

Whether the sorting of the Turkish immigrants is large enough to bias our estimates is a hard question to answer. ${ }^{24}$ To ensure that this is not a major issue, we conducted two additional tests that exclude areas which are potentially subject to the endogeneity bias created by the sorting of the Turkish immigrants. First, we exclude from the sample the 22 RORs where Turkish immigrants are more likely to migrate to and from. We identified these through the data we used in the regression in Table 4. We recognize that this is a rather blunt approach, which might not solve directly the endogeneity issue. It may also create some distortion, given that we implicitly exclude from the analysis also natives and immigrant groups which might be exempt from sorting issues. However, we believe that this test is helpful to provide an idea of how large the sorting issue could be. Estimates of the baseline model show that the ED index coefficient is 0.969 (s.e. 0.178). In the second test, we take a radical approach and exclude the top ten RORs with the highest SWB from the sample. ${ }^{25}$ If areas with high SWB work as a catalyzer for Turkish immigrants, and if the sorting is serious, then our results could be driven by these areas. Instead, we find that the estimate of the ED index is still large and strongly significant (0.571, s.e. 0.176). The results from these two tests can be considered as bounds of our baseline estimates. Overall, our results still hold in the conservative scenario in which we exclude areas that could be affected by the endogenous sorting of the largest ethnic group.

Instrumenting Ethnic Diversity. The analysis so far has demonstrated that self-selection is not likely to substantially affect our fixed-effects estimates, making us thinking that we are "close to" a causal interpretation. Indeed, in order to obtain a true causal estimate, one would need either exogenous variation in ethnic diversity or a valid instrument. The search

\footnotetext{
${ }^{23}$ Interestingly, the propensity of internal migration is fairly similar across ethnic groups (being 0.13 on average).

${ }^{24}$ Ideally and to be more rigorous, one would calculate an ED index that excludes all immigrants who migrate internally. This is not feasible in our case, since the only possible way of obtaining estimates of mobility of immigrants is via the GSOEP which - due to the small sample size of immigrants who move would produce imprecise predictions to be used to correct the official statistics we use in this paper.

${ }^{25}$ The top ten SWB RORs are: Oberfranken-Ost, Schleswig-Holstein Mitte, Paderborn, Hamburg, Osnabrueck, Bodensee-Oberschwaben, Ostwuerttemberg, Oberland, Bremen and Rheinpfalz.
} 
Table 4: Germans out-migration and immigrant sorting

\begin{tabular}{|c|c|c|c|c|c|c|c|c|c|}
\hline & \multicolumn{3}{|c|}{ Germans out-migration } & \multicolumn{5}{|c|}{ Immigrant sorting } & \multirow[b]{2}{*}{ IX } \\
\hline & I & II & III & IV & $\mathrm{V}$ & VI & VII & VIII & \\
\hline Ethnic diversity (d) - (o) & $\begin{array}{l}-0.4582 \\
(0.4260)\end{array}$ & $\begin{array}{l}-0.3802 \\
(0.4299)\end{array}$ & $\begin{array}{l}-0.2751 \\
(0.4351)\end{array}$ & $\begin{array}{l}-1.1103 \\
(1.0684)\end{array}$ & $\begin{array}{r}-1.1160 \\
(1.0638)\end{array}$ & $\begin{array}{l}-1.8352 \\
(1.1532)\end{array}$ & $\begin{array}{r}-0.9470 \\
(1.0879)\end{array}$ & $\begin{array}{l}-0.9476 \\
(1.0848)\end{array}$ & $\begin{array}{l}-1.9395^{*} \\
(1.1327)\end{array}$ \\
\hline Immigrant share (d) - (o) & & $\begin{array}{c}0.0094^{*} \\
(0.0054)\end{array}$ & $\begin{array}{r}0.0064 \\
(0.0095)\end{array}$ & & $\begin{array}{l}-0.0053 \\
(0.0136)\end{array}$ & $\begin{array}{l}-0.0324 \\
(0.0237)\end{array}$ & & $\begin{array}{r}-0.0030 \\
(0.0129)\end{array}$ & $\begin{array}{l}-0.0404^{*} \\
(0.0223)\end{array}$ \\
\hline Unemployment rate $(\mathrm{d})-(\mathrm{o})$ & & & $\begin{array}{l}-0.0159^{* * *} \\
(0.0058)\end{array}$ & & & $\begin{array}{r}0.0033 \\
(0.0172)\end{array}$ & & & $\begin{array}{l}-0.0007 \\
(0.0174)\end{array}$ \\
\hline Per capita log GDP (d) - (o) & & & $\begin{array}{r}0.0276 \\
(0.1512)\end{array}$ & & & $\begin{array}{r}0.5604 \\
(0.4061)\end{array}$ & & & $\begin{array}{l}0.7782^{* *} \\
(0.3838)\end{array}$ \\
\hline $\operatorname{SWB}(\mathrm{d})-(\mathrm{o})$ & & & & $\begin{array}{r}0.1032 \\
(0.1690)\end{array}$ & $\begin{array}{r}0.1152 \\
(0.1740)\end{array}$ & $\begin{array}{r}0.0564 \\
(0.1964)\end{array}$ & $\begin{array}{l}-0.4108 \\
(0.5821)\end{array}$ & $\begin{array}{l}-0.4095 \\
(0.5826)\end{array}$ & $\begin{array}{l}-0.5821 \\
(0.6025)\end{array}$ \\
\hline SWB $(d)-(o) \times$ Turkey & & & & & & & $\begin{array}{l}1.7744^{* * *} \\
(0.6568)\end{array}$ & $\begin{array}{l}1.7742^{* * *} \\
(0.6578)\end{array}$ & $\begin{array}{l}1.9861^{* * *} \\
(0.6852)\end{array}$ \\
\hline SWB (d) $-(o) \times$ Russia & & & & & & & $\begin{array}{r}0.6126 \\
(0.7857)\end{array}$ & $\begin{array}{r}0.6182 \\
(0.7904)\end{array}$ & $\begin{array}{r}0.8179 \\
(0.8021)\end{array}$ \\
\hline SWB $(d)-(o) \times$ Poland & & & & & & & $\begin{array}{r}0.9373 \\
(0.7084)\end{array}$ & $\begin{array}{r}0.9375 \\
(0.7058)\end{array}$ & $\begin{array}{r}0.9257 \\
(0.7191)\end{array}$ \\
\hline SWB (d) - (o) $\times$ Ex-Yugoslavia & & & & & & & $\begin{array}{r}0.3576 \\
(0.7560)\end{array}$ & $\begin{array}{r}0.3721 \\
(0.7547)\end{array}$ & $\begin{array}{r}0.4957 \\
(0.7486)\end{array}$ \\
\hline SWB $(d)-(o) \times$ Kazakhstan & & & & & & & $\begin{array}{r}0.0259 \\
(0.7504)\end{array}$ & $\begin{array}{r}0.0302 \\
(0.7516)\end{array}$ & $\begin{array}{r}-0.0953 \\
(0.7462)\end{array}$ \\
\hline SWB $(d)-(o) \times$ Other & & & & & & & $\begin{array}{r}0.2695 \\
(0.6144)\end{array}$ & $\begin{array}{r}0.2776 \\
(0.6162)\end{array}$ & $\begin{array}{r}0.3355 \\
(0.6319)\end{array}$ \\
\hline$R^{2}$ & 0.027 & 0.030 & 0.037 & 0.051 & 0.052 & 0.061 & 0.091 & 0.091 & 0.109 \\
\hline $\mathrm{N}$ & 18,097 & 18,097 & 18,097 & 1,684 & 1,684 & 1,684 & 1,684 & 1,684 & 1,684 \\
\hline
\end{tabular}

for instruments for migration-related variables has preoccupied scholars for years. The most convincing instrumental variable approach so far has been to employ as instrument either unexpected events that cause large migrations, such as the conflict in former Yugoslavia (e.g., Angrist and Kugler, 2003) or the fall of the Berlin Wall (e.g., D'Amuri et al., 2010), or to exploit the role of policy, such as the Swedish initiative involving a random allocation of refugees (e.g, Edin et al., 2003). These instruments provide a very convincing estimate for the local average treatment effect, but their external validity is usually limited to the time/year of the exogenous event. With a long period of time like the one in our data, it is unlikely that a single event - even if substantial - would provide variation for all 15 years. In absence of such exogenous variation, the typical solution is to rely on lags of the endogenous variable (see e.g., Hatton and Tani, 2005). In our particular case, the argument would be that past ethnic diversity is less likely to be affected by contextual unobserved shocks especially after having controlled for unobservable regional heterogeneity, and annihilating the role of local- and year-specific shocks by including time-varying controls such as the local unemployment rate.

The IV regression produces an estimate somewhat lower (0.676, s.e. 0.224$)$ than the 
one estimated in the benchmark model, but still large and statistically significant. ${ }^{26} \mathrm{~A}$ lower estimate is compatible with an upward bias of the fixed-effect model. This could be determined, for example, by the presence of time-varying unobservable factors that could positively and simultaneously affect diversity and natives' well-being in one area. However, under the assumption that the instrument is valid, it seems that the role of such unobservable factors is marginal and does not substantially affect our overall result.

\subsection{Who is Affected?}

The estimated welfare "gains" are an average effect. In this subsection, we investigate the heterogeneity of the relationship between ethnic diversity and well-being with respect to individual characteristics of Germans. To do so, we divide our sample in subgroups and perform separate regressions. In order to not induce a biased split of the data, we select characteristics that are as exogenous as possible or relatively stable over the period of analysis. The dimensions that we consider are sex, age, education, marital status and personality traits. All regressions are estimated using our preferred fixed-effects specification. The results are summarized in Table 5, where we only report the estimate for the ED index.

Panel A of Table 5 shows the results for different socio-demographic groups. Males are slightly more satisfied with ethnic diversity than females, although our tests reveal that coefficients are not statistically different at usual significance levels. ${ }^{27}$ The age pattern is much clearer: The effect gradually decreases as individuals get older and is essentially zero for those older than 50. While the estimates for the groups "younger than 35 years" and "aged 35-50" are not statistically different from each other, they are both quantitatively and statistically different with respect to the group "older than 50 years" (p-values are 0.035 and 0.051, respectively). Next, we investigate marital status. The effect is stronger among single people, although the estimate is borderline statistically different from the group of natives who are married $(\mathrm{p}$-value $=0.12)$. Lastly, we consider three education categories (roughly corresponding to terciles of the education distribution). The estimated effect is stronger for less educated Germans. Our tests show no difference between the groups with low and intermediate education, but the estimates of both groups are statistically different from the group with high education (p-values are 0.049 and 0.047 , respectively).

\footnotetext{
${ }^{26}$ The estimate of the lagged ethnic diversity in the first stage is 0.675 (s.e. 0.002 ). The $F$-stat is 119,912 and the partial $R^{2}$ is 0.474 . Full estimation results can be obtained from the authors upon request.

${ }^{27}$ To test the statistical difference of the estimates, we pool the various groups (e.g., males and females), create an indicator for the group (e.g., a male dummy) and estimate a fully interacted model where we interact the group indicator with all explanatory variables. We test the null hypothesis that the interaction between the group indicator and the ED index is equal to zero. If this is not rejected, the estimates are not statistically different from each other.
} 


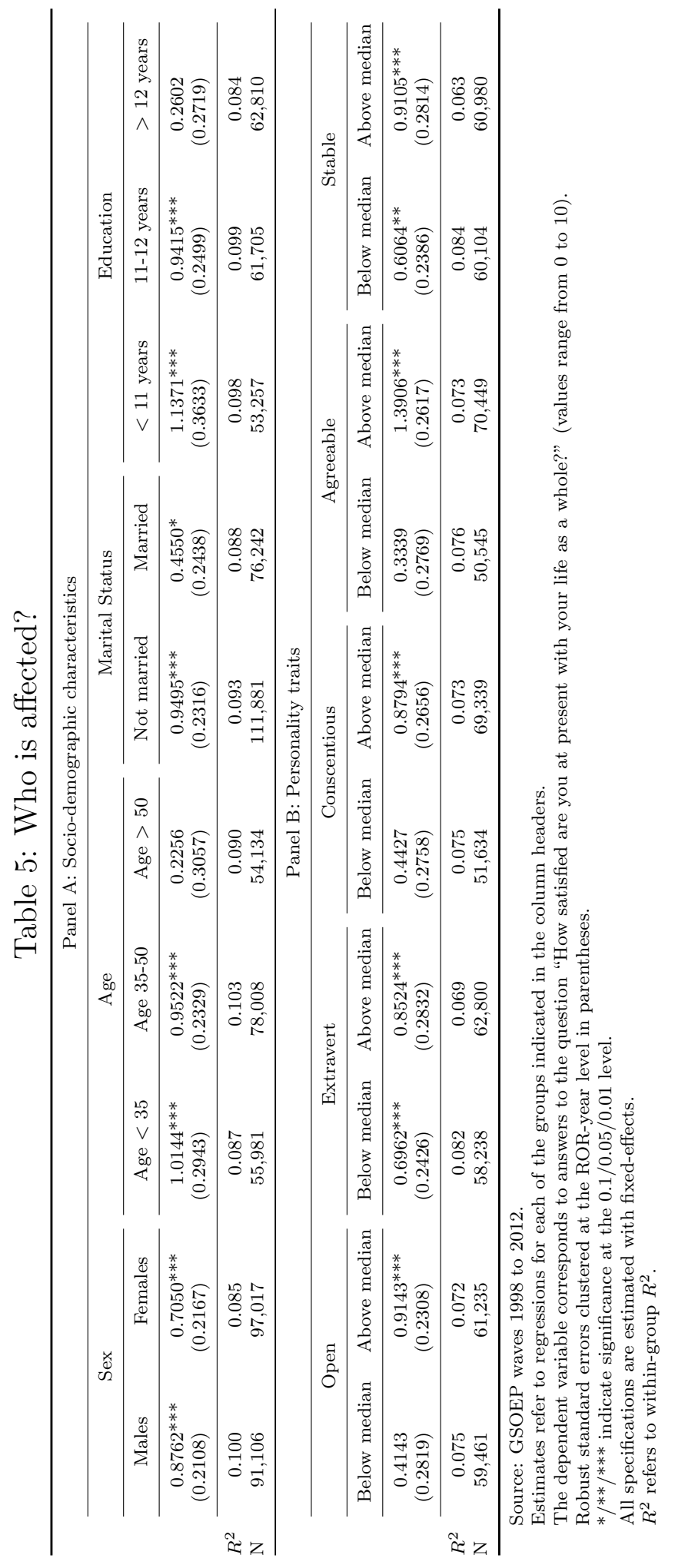


Panel B of Table 5 shows the results where we split the sample according to the personality traits of Germans. For the scope of this study, we use measures of psychological traits known as the "Big Five". These are measures constructed using responses to questions about personality attributes (see e.g., Costa and McCrae, 1992, Goldberg, 1992). The GSOEP contains a 15-item inventory on the basis of which the Big Five are constructed (for details, see Gerlitz and Schupp, 2005). These are: conscientiousness, extraversion, agreeableness, openness, and neuroticism. We coded the variables in a way that a higher value indicates a more "positive" trait. We reverse the scale of neuroticism, so a higher value means higher emotional stability (i.e., less neuroticism). Data are collected only periodically and so far in the waves 2005, 2009 and 2013. In order to maximize the sample size, we make the assumption that personality traits are stable within individuals, at least in the medium run. Given this, we "extend" the values of the personality traits for the three years preceding each of the three data points in which they are observed. Hence, for each individual, we use the 2005 value for years 2002, 2003, 2004 and 2005, and the 2009 value for years 2006, 2007, 2008 and 2009. We also exploit data from 2013 (which are not used since regional statistics for that year are still not published) to obtain values for 2010, 2011 and 2012. The sample size for this test is smaller than the benchmark sample, but still large enough to conduct panel analysis. Exploiting the stability of the Big Five over the medium period does not seem an unreasonable conjecture, given that these traits have been proven to be stable within a four-year period in a study using the Household, Income and Labour Dynamics in Australia (Cobb-Clark and Schurer, 2012), a dataset with personality questions similar to GSOEP.

Once obtained the five traits, we split the sample in two parts: individuals with high and low values of each trait, depending on whether the reported value is above or below the median of the sample. This allows us to explore the extent to which the effect of ethnic diversity on SWB varies according to personality. Remarkably, the results show that the estimated effect is larger for natives with higher values of the Big Five traits. Although the estimates for the "high" and "low" type can be said to be statistically different only in the case of agreeableness ( $\mathrm{p}$-value $<0.01$ ), the pattern of the results consistently shows that ethnic diversity has a larger effect for natives who are open to experience and are self-disciplined, have positive emotions and a cooperative character, as well as they are less anxious.

\section{Where is the Result Coming From?}

After having established that the estimated effect of ethnic diversity on SWB is robust to several confounding factors, we investigate the possible mechanisms behind our results. 
While we acknowledge that there could be many explanations, we focus on two main channels: productivity/skills and social capital. These are somewhat different channels that could be at work separately or simultaneously.

The literature has identified that ethnic diversity might affect labor productivity, since a diverse set of skills brought by immigrants can generate positive externalities. The presence of such productivity gains could itself improve the well-being of firms (through their overall performance) and of consumers (by reducing prices or increasing the basket of available commodities, e.g., ethnic goods/services). Hence, the positive effect that we estimated would be an indirect consequence of these economic spillovers. However, it is also possible that there are other channels beyond the economic sphere. For example, higher ethnic diversity could affect trust and civic participation of the host population and, in turn, their well-being.

We acknowledge that it is hard to isolate the role of all channels. Notwithstanding this, we attempt to empirically assess the importance of the two mechanisms above by performing regression analyses where we interact the ED index with an indicator that is thought to proxy productivity or social capital. When statistically significant, we interpret these two variables as "mediators" of the relationship between ethnic diversity and natives' SWB. Defining $D$ as the indicator of interest, we modify equation (1) as follows:

$$
\begin{aligned}
S W B_{i t}^{*} & =\beta_{0} D_{i}+\beta_{1} D_{i} \times E D_{r t}+\beta_{2}\left(1-D_{i}\right) \times E D_{r t}+\phi I M_{r t}+\mathbf{Z}_{r t}^{\prime} \boldsymbol{\lambda}+\mathbf{X}_{i t}^{\prime} \gamma+\varepsilon_{i t} \\
\varepsilon_{i t} & =\rho_{r}+\tau_{t}+\alpha_{i}+\nu_{i t}
\end{aligned}
$$

Note that in (2) we have re-parametrized the coefficients in a way that we do not have a main effect for ED, but two interaction terms (when $D=1$ and $D=0$ ), which allows us comparing the ethnic diversity effect depending on the value of the indicator. Tables 6 and 7 report only the estimates of these interaction terms.

\subsection{Productivity and Skills}

Productivity Gains. One important finding in the recent literature is that ethnic diversity has productivity benefits (e.g., Audretsch and Feldman, 1996, Glitz, 2014, Suedekum et al., 2014, Trax et al., 2015). Higher ethnic diversity implies a more diversified set of skills and ability, and hence a positive externality for employers, workers, and consumers. In order to capture this channel, we use labor supply (working hours and employment status), wage

and household income of Germans as proxy for productivity. We characterize $D=1$ the status for which individuals have a value of these variables above the median and $D=0$ otherwise. The results are presented in the first four columns of the top panel of Table 
6. We can see that the welfare effect of ethnic diversity is slightly larger for natives with higher wage and longer working hours, while it is the same irrespective of the employment status. However, a t-test for the equality of the interactions above and below the median cannot reject the null hypothesis that these are statistically identical for all three measures of productivity.

When we investigate household income we find that the effect is substantially stronger for less wealthy Germans. The estimates for the two interaction terms are statistically different from each other (the p-value is 0.028 ). One potential interpretation is that when productivity (proxied by income) is already high, ethnic diversity can only marginally improve it. When productivity is lower, ethnic diversity (which arguably enriches the set of skills and ability available in the market) leads to larger productivity gains and thereby a higher level of well-being.

Local Markets. Next, we investigate the productivity channel by moving the dimension from the individual to the local labor market level. We construct the interactions by creating an indicator for high and low productive RORs by using macroeconomic variables such as regional GDP, unemployment and the immigrant share. The results are reported in the last three columns of the top panel of Table 6. The effect is stronger for less productive areas (low GDP and high unemployment), with the estimates of the interaction terms in both cases being statistically different at the $10 \%$ significance level (p-values are 0.082 and 0.051, respectively). This pattern is consistent with the result found for the income at the individual level. When we focus on the immigrant share, we find no effect for highimmigration regions, and a strong effect for low-immigration RORs (the p-value for difference between the two interactions is 0.021). Since immigrants sort into highly productive and richer areas (e.g., because of more job opportunities), it is not surprising that the result for high-immigration areas is similar to the one for high-income areas. This result complements the one in column III of Table 2, where we found that ethnic diversity and immigrant share work as "substitutes". While it is difficult to attach a causal interpretation to these interaction terms, the results suggest that the effect of ethnic diversity is stronger in less productive areas.

Ethnic Diversity or Immigrants' Skills? The final dimension that we consider within the productivity channel has to do with the composition of immigrants, in particular with respect to their skills. We first consider their education composition. In order to do so, we pool GSOEP data and calculate the average education level of immigrants in each ROR. This allows us to derive an indicator for RORs with high versus low skills of immigrants. 
The first column in the bottom panel of Table 6 presents the results. The welfare gain of ethnic diversity is stronger in regions where education is higher, but the estimates of the two interactions are not statistically different from each other. On the other hand, the pattern of the results is much clearer when we characterize RORs in terms of German language skills. Consistently, the effect is stronger for Germans who live in RORs where immigrants have better language skills. When language skills are measured by the self-assessed level of spoken German or by whether immigrants speak German at home, the estimates of the interactions are statistically different from each other (p-values are $<0.01$ and 0.024 , respectively). In the case of written German, the estimate for the above mean interaction is still higher but not statistically different from the below mean interaction. These results suggest that natives' gains from interacting with other cultures (and possibly languages) are higher when immigrants acquire German language skills and a better understanding of the local culture, likely because these promote communication and social interactions.

We further delve into this point by looking at how immigrants are assimilated. In particular, we constructed measures using responses to questions about how much immigrants "feel German" and "feel foreign" to indicate the attachment to the host or home country (high levels of feeling foreign can be thought of capturing low levels of cultural assimilation). This allows us to obtain the average level of immigrant assimilation in the region. We then derive indicators for whether the ROR where the natives live is above or below the median value of the two indicators. Remarkably, the effect is stronger for Germans who live in RORs where immigrants are more assimilated. For both measures, the interaction term between ethnic diversity and the indicator for high assimilated region is statistically different from the interaction with the indicator for low assimilated RORs ( $p$-values are $<0.01$ with the measure "feel German" and 0.01 with the measure "feel foreign"). The effect of diversity is in fact negative for RORs where immigrants do not feel German. We find a similar pattern also when we consider a measure of economic assimilation such as wages. However the interaction terms are not statistically different from each other. ${ }^{28}$

Overall, immigrants' skills appear to be a potential channel for explaining our results, especially those related to the socio-cultural assimilation of immigrants in Germany. Higher diversity implies that no immigrant group constitutes the "majority" in a given region, and in general immigrants do not segregate into enclaves. This makes the use of German lan-

\footnotetext{
${ }^{28}$ We constructed the wage assimilation measure following Akay et al. (2014) closely. In practice, using GSOEP, we perform wage regressions separately for natives and immigrants. We then predict the wages for the two groups and calculate averages for each ROR. Finally, we construct the ratio between the average predicted wages of immigrants and that of natives. Values of the ratio below 1 indicate lower wage assimilation, while values of 1 or larger a higher level of assimilation. Finally, we derive the indicator for values above and below the median of the assimilation ratio.
} 
guage more diffused among immigrants. In turn, language uniformity increases economic interactions between Germans and immigrants allowing for reciprocal spillovers (e.g., immigrants can increase their job opportunities; Germans can have access to a more diverse set of skills, consumption goods, networks etc.). The socio-cultural assimilation implied by diversity, and its potential productivity effects are also compatible with what we found with the decomposition of the Shannon Entropy index: well-being gains are higher in more integrated areas.

Table 6: Productivity and skills

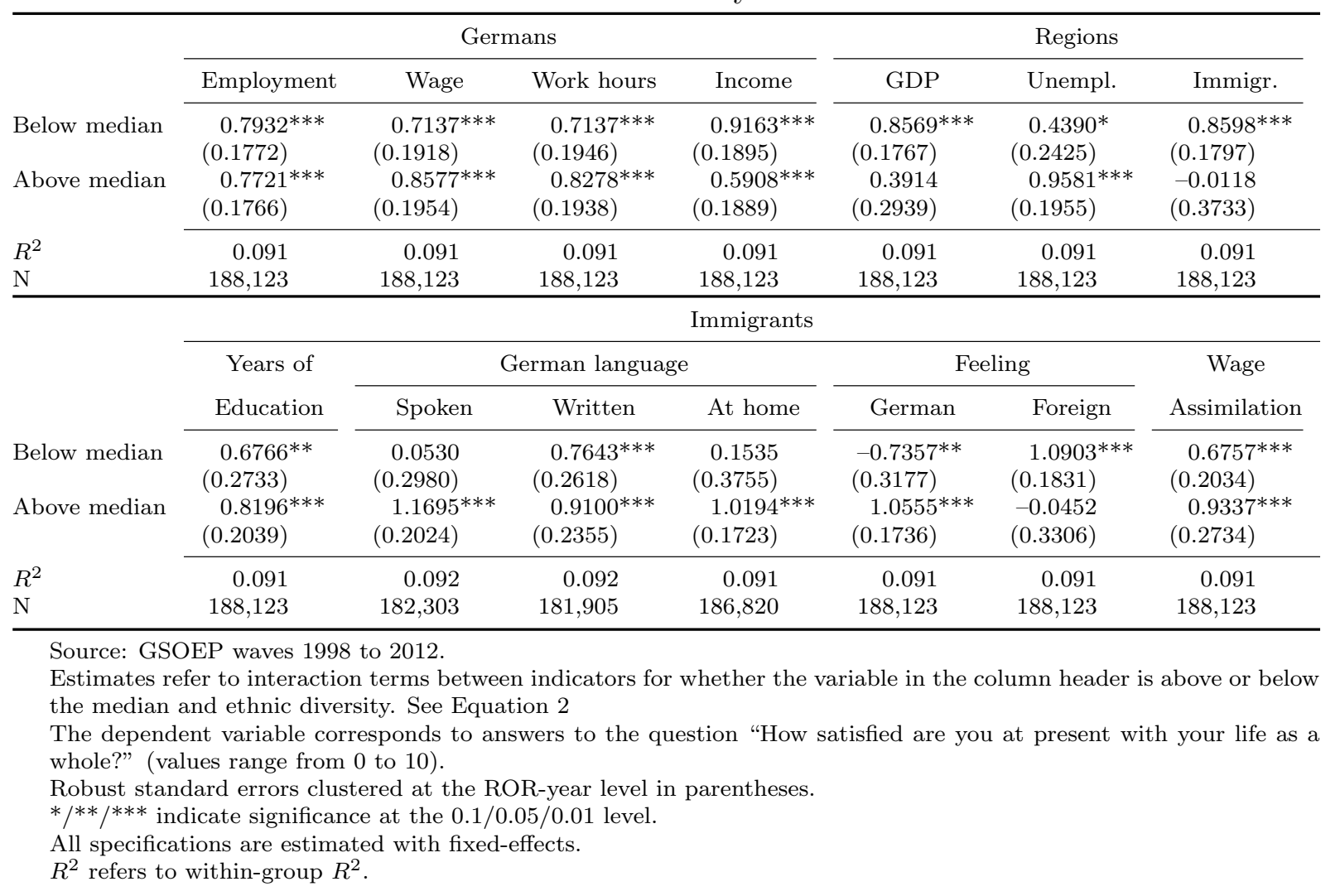

\subsection{Social Capital}

Trust and Civic Engagement. In this subsection, we investigate whether natives' trust is a mediating factor between ethnic diversity and well-being. Trust is a relevant measure of social capital in our context because of its known influence on well-being (Putnam, 2007, Helliwell et al., 2009, Winkelmann, 2009). At the same time, diversity might, on one hand generate ethnolinguistic and cultural barriers to trust formation, and on the other hand create an incentive for cooperation and civic participation among members of the groups. ${ }^{29}$

\footnotetext{
${ }^{29}$ The trust questions have been experimentally validated (see Fehr et al., 2003, for more details).
} 
We derive a measure of trust by summing the values of the three variables present in the GSOEP. These correspond to answers to the following statements: "On the whole one can trust people", "Nowadays one cannot rely on anyone", and "If one is dealing with strangers, it is better to be careful before one can trust them". ${ }^{30}$ We then create an indicator for whether the level of trust of the individual is high (i.e., above median), and interact it with the ethnic diversity variable. Results are presented in the first three columns of Table 7. The estimates show that the effect of diversity on SWB is stronger for individuals with lower level of trust. However, t-test for the difference of the estimates that we computed for the three regression models reveal that the interaction terms are not statistically different from each other. This weak statistical result does not allow us to conclude that the gains from interacting with immigrants could be larger for less trustworthy individuals. ${ }^{31}$

Another dimension we investigate is civic engagement. We follow the definition used in the GSOEP study by Hener et al. (2016), who define an index of civic engagement as the aggregation of four components: political interest, party identification, organizational involvement and individual voluntarism. Interest in politics and party identification are in general associated with involvement in public matters. Similarly, organization membership and volunteering are also forms of active public participation. We construct an indicator for whether Germans have a level of civic engagement above or below the median and interact it with the ED index. The estimates in the fourth column of Table 7 indicate that there are no differences depending on the level of civic engagement. Hence, it could be that ethnic diversity does not interact with civic engagement, or that it does in the same way for individuals who are interested in public matters and those who are not.

Cultural Interactions and Multiculturality. A final dimension is multiculturality, defined as the presence of a variety of cultures. In general, ethnic diversity is thought to generate a multicultural environment to the extent that ethnic groups mix and interact, instead of segregating into enclaves. We investigate whether a multicultural environment is a mechanism behind our results. To do so, we use information on cultural activities attended by natives and on tourism at the regional level. We construct an indicator for whether Germans often attend cultural events (the indicator takes the value of 1 for those who attend at

\footnotetext{
${ }^{30}$ Respondents can agree or disagree (on a 4-point scale, higher values correspond to stronger agreement). We harmonize the scales so that higher values mean strong agreement to the first question and strong disagreement to the second and third. We obtain similar results if we perform principal component analysis. Since trust questions are asked only in 2003 and 2008, we use only data that refer to these two periods.

${ }^{31}$ It is possible that there is a causal relationship between ethnic diversity and trust. While this has been found in some studies (e.g., Putnam, 2007), it does not seem of particular concern in our case, since the correlations between the three measures of trust with ethnic diversity are all small and insignificant (-0.005 (s.d. 0.406), 0.001 (s.d. 0.920), and 0.002 (s.d. 0.692), respectively).
} 
least once a month) and interact it with the ethnic diversity variable. Results in column $\mathrm{V}$ of Table 7 reveal that the effect of ethnic diversity is higher for individuals who attend more events. The estimates of the two interaction terms are significantly different from each other (p-values <0.01). One possible explanation is that individuals who often attend cultural events have also more social interactions and larger networks which in turn could increase the positive effects of ethnic diversity.

Table 7: Social capital

\begin{tabular}{lcccc}
\hline & $\begin{array}{c}\text { Trust } \\
\text { people }\end{array}$ & $\begin{array}{c}\text { Civic } \\
\text { engagement }\end{array}$ & $\begin{array}{c}\text { Cultural } \\
\text { events }\end{array}$ & Tourism \\
\cline { 2 - 5 } Below median & $0.9818^{* *}$ & $0.6294^{* * *}$ & $0.5967^{* * *}$ & $0.5382^{* * *}$ \\
& $(0.4641)$ & $(0.2265)$ & $(0.2252)$ & $(0.2015)$ \\
Above median & 0.6483 & $0.6618^{* *}$ & $0.7066^{* * *}$ & $0.9682^{* * *}$ \\
& $(0.4142)$ & $(0.2611)$ & $(0.2242)$ & $(0.2261)$ \\
\cline { 2 - 5 }$R^{2}$ & 0.114 & 0.093 & 0.094 & 0.091 \\
$\mathrm{~N}$ & 26,510 & 106,717 & 107,635 & 188,123 \\
\hline
\end{tabular}

Source: GSOEP waves 1998 to 2012.

Estimates refer to interaction terms between indicators for whether the variable in the column header is above or below the median and ethnic diversity. See Equation 2

The dependent variable corresponds to answers to the question "How satisfied are you at present with your life as a whole?" (values range from 0 to 10$)$.

Robust standard errors clustered at the ROR-year level in parentheses. $* / * * / * * *$ indicate significance at the $0.1 / 0.05 / 0.01$ level.

All specifications are estimated with fixed-effects.

$R^{2}$ refers to within-group $R^{2}$.

We obtained a similar result when we use a measure of tourism intensity. This is constructed using information on the number of overnight stays in touristic facilities in the ROR (e.g., hotels) obtained from the INKAR data. We construct an indicator for whether the region has a touristic intensity above or below the median and interact it with the ED index. The effect of ethnic diversity is stronger for natives who live in more touristic areas, with the difference of the two interaction terms being significant at the $10 \%$ level (p-value 0.082). One possible interpretation is that touristic regions might be a more favorable environment for immigrants to establish businesses and could create positive externalities for the natives living in these areas, for example by providing a larger supply of ethnic goods and services.

\section{Conclusion}

Ethnic diversity has been increasing in many developed countries in the past decades. In this paper, we investigate the relationship between the ethnic diversity of immigrants and 
the well-being of natives in Germany. One of the novelties of this study is that it brings several data sources together. The main data are from the GSOEP's waves from 1998 to 2012, which have been merged with regional immigration data available with the split by immigrant nationalities. These combined longitudinal data allow us to control for unobserved heterogeneity at the individual, regional and time level.

We find several important results. First, the correlation between ethnic diversity and Germans' well-being is positive. That is, there is a welfare gain from higher diversity. This result is found to be robust with respect to several checks with estimators and ethnic diversity measures. However, we also find that it is the diversity within each region that positively correlates with the well-being, but not the diversity across areas: natives' well-being is relatively lower when they live in ethnically segregated enclaves. We then investigate the causal interpretation of the results, finding that the spatial redistribution of natives and immigrants across regions does not confound the results.

We explored productivity and social capital as potential channels behind our results. There is weak evidence that ethnic diversity enhances the productivity of natives. On the other hand, we found that the socio/economic assimilation of immigrants in the region is important. When we consider social capital, we found that both trust and cultural interactions are relevant channels, albeit only in the second case are results statistically significant. We are aware that these explanations are not exhaustive, however they cover economic and social aspects of daily life which are likely to interact with ethnic diversity. Moreover, they complement our evidence that the effect of ethnic diversity is stronger when more weight is given to groups of immigrants that are culturally and economically closer to Germans.

Migration is a complex phenomenon that influences many aspects of life. Our study delves for the first time into the influence of ethnic diversity on human well-being and allows us to better understand how the size and composition of immigrants affect overall utility. That diversity is beneficial to people is perhaps not so surprising, given that it is embedded into the evolution of the human species. What we think our analysis is capturing are the externalities created by the interaction of complementary sets of skills, cultures and traditions pertaining to each ethnic group. Such interaction creates economic and social spillovers that are not only beneficial to the immigrant groups, but to the host population as well. Coupled with the results of the seminal study by Akay et al. (2014), our finding suggests that both the level of immigration and its diversity positively influence well-being.

Our evidence is limited to Germany for the period under analysis. With its traditional role in attracting large and diverse waves of immigrants, Germany represents an interesting case study. At the same time, it is known that the recent economic crisis affected the German labor market less than other countries. It is possible that the relationship between well-being 
and ethnic diversity is influenced by the economic cycle - irrespective of the real economic impact of migration. Hence results could be different in areas that were particularly affected by the economic crisis. It is difficult to generalize our results to other countries, because of the differences in terms of migration history, the size and composition of immigrants and their socio-economic integration. For example, the results of the Brexit vote, and the fact that immigration was a central aspect in the debate is the reflection that a large share of the British voters became averse to immigration and/or immigrants. We hope that our approach can motivate future work, which could extend our research question to other countries or dig further into the mechanisms linking ethnic diversity and well-being. 
The authors declare that they have no conflict of interest. 


\section{References}

Akay, A., O. Bargain, and K. F. Zimmermann (2016). Home Sweet Home? Macroeconomic Conditions in Home Countries and the Well-Being of Migrants. Journal of Human Resources, forthcoming.

Akay, A., A. Constant, and C. Giulietti (2014). The Impact of Immigration on the Well-being of Natives. Journal of Economic Behavior and Organization 103, 72-92.

Akay, A. and P. Martinsson (2009). Sundays Are Blue: Aren't They? The Dayof-the-Week Effect on Subjective Well-Being and Socio-Economic Status. IZA Discussion Papers 4653.

Akay, A. and P. Martinsson (2012). Positional Concerns through the Life Cycle: Evidence from Subjective Well-being Data and Survey Experiments. IZA Discussion Papers 6342.

Alesina, A., R. Baqir, and W. Easterly (1999). Public Goods and Ethnic Divisions. Quarterly Journal of Economics 114(4), 1243-1284.

Alesina, A., J. Harnoss, and H. Rapoport (2016). Birthplace Diversity and Economic Growth. Journal of Economic Growth 21, 101-138.

Alesina, A. and E. La Ferrara (2002). Who Trusts Others? Journal of Public Economics 85(2), 207-234.

Angrist, J. D. and A. D. Kugler (2003). Protective or Counter-Productive? Labour Market Institutions and the Effect of Immigration on EU Natives. The Economic Journal 113(488), F302-F331.

Audretsch, D. B. and M. P. Feldman (1996). R\&D Spillovers and the Geography of Innovation and Production. The American Economic Review 86(3), 630-640.

Baetschmann, G., K. E. Staub, and R. Winkelmann (2015). Consistent Estimation of the Fixed Effects Ordered Logit Model. Journal of the Royal Statistical Society: Series A (Statistics in Society) 178(3), 685-703.

Blanchflower, D. G. and A. J. Oswald (2008). Is Well-being U-shaped over the Life Cycle? Social Science 83 Medicine 66(8), 1733-1749.

Borjas, G. J. (2000). Ethnic Enclaves and Assimilation. Swedish Economic Policy Review 7(2), 89-122. 
Boyce, C. J., A. M. Wood, and G. D. Brown (2010). The Dark Side of Conscientiousness: Conscientious People Experience Greater Drops in Life Satisfaction Following Unemployment. Journal of Research in Personality 44(4), 535-539.

Brunow, S. and U. Blien (2014). Effects of Cultural Diversity on Individual Establishments. International Journal of Manpower 35(1-2), 166-186.

Brunow, S. and P. Nijkamp (2012). The Impact of a Culturally Diverse Workforce on Firms' Market Size: An Empirical Investigation on Germany. Norface Discussion Papers 22.

Clark, A. E. and A. J. Oswald (1994). Unhappiness and Unemployment. The Economic Journal 104(424), 648-659.

Cobb-Clark, D. A. and S. Schurer (2012). The Stability of Big-Five Personality Traits. Economics Letters 115(1), 11-15.

Constant, A., L. Gataullina, and K. F. Zimmermann (2009). Ethnosizing Immigrants. Journal of Economic Behavior and Organization 69(3), 274-287.

Constant, A. and D. S. Massey (2003). Self-selection, Earnings, and Out-migration: A Longitudinal Study of Immigrants to Germany. Journal of Population Economics 16(4), 631-653.

Constant, A. and K. F. Zimmermann (2008). Measuring Ethnic Identity and Its Impact on Economic Behavior. Journal of the European Economic Association 6(2-3), 424-433.

Costa, P. T. and R. R. McCrae (1992). Normal Personality Assessment in Clinical Practice: The NEO Personality Inventory. Psychological Assessment 4(1), 5.

D'Amuri, F., G. I. Ottaviano, and G. Peri (2010). The Labor Market Impact of Immigration in Western Germany in the 1990s. European Economic Review 54(4), 550-570.

Diener, E., E. Suh, R. E. Lucas, and H. L. Smith (1999). Subjective Well-being: Three Decades of Progress. Psychological Bulletin 125(2), 276-302.

Dolan, P., T. Peasgood, and M. White (2008). Do we Really Know what Makes us Happy? A Review of the Economic Literature on the Factors Associated with Subjective Well-being. Journal of Economic Psychology 29(1), 94-122.

Edin, P.-A., P. Fredriksson, and O. Åslund (2003). Ethnic Enclaves and the Economic Success of Immigrants. Evidence from a Natural Experiment. The Quarterly Journal of Economics 118(1), 329-357. 
Fehr, E., U. Fischbacher, B. von Rosenbladt, J. Schupp, and G. G. Wagner (2003). A Nation-Wide Laboratory: Examining Trust and Trustworthiness by Integrating Behavioral Experiments into Representative Surveys. IZA Discussion Papers 715.

Ferrer-i Carbonell, A. (2005). Income and Well-being: An Empirical Analysis of the Comparison Income Effect. Journal of Public Economics 89(5), 997-1019.

Ferrer-i Carbonell, A. and P. Frijters (2004). How Important is Methodology for the Estimates of the Determinants of Happiness? The Economic Journal 114(497), 641-659.

Frey, B. S. and A. Stutzer (2002). What can Economists Learn from Happiness Research? Journal of Economic literature 40(2), 402-435.

Frijters, P., J. P. Haisken-DeNew, and M. A. Shields (2004). Investigating the Patterns and Determinants of Life Satisfaction in Germany Following Reunification. Journal of Human Resources 39(3), 649-674.

Gerlitz, J.-Y. and J. Schupp (2005). Zur Erhebung der Big-Five-basierten Persoenlichkeitsmerkmale im SOEP. DIW Research Notes 4.

Glitz, A. (2014). Ethnic Segregation in Germany. Labour Economics 29, 28-40.

Goldberg, L. R. (1992). The Development of Markers for the Big-Five Factor Structure. Psychological assessment 4 (1), 26.

Graham, C. and J. R. Pozuelo (2017). Happiness, Stress, and Age: How the U-Curve Varies across People and Places. Journal of Population Economics fortchoming.

Hatton, T. J. and M. Tani (2005). Immigration and Inter-Regional Mobility in the UK, 1982-2000. The Economic Journal 115(507), F342-F358.

Helliwell, J. F., C. P. Barrington-Leigh, A. Harris, and H. Huang (2009). International Evidence on the Social Context of Well-Being. NBER Working Paper Series, 14720.

Hener, T., H. Rainer, and T. Siedler (2016). Political Socialization in Flux?: Linking Family non-Intactness during Childhood to Adult Civic Engagement. Journal of the Royal Statistical Society: Series A (Statistics in Society), forthcoming.

Hewlett, S. A., M. Marshall, and L. Sherbin (2013). How Diversity can Drive Innovation. Harvard Business Review 91 (12), 30-30. 
Jaeger, D. A., T. Dohmen, A. Falk, D. Huffman, U. Sunde, and H. Bonin (2010). Direct Evidence on Risk Attitudes and Migration. The Review of Economics and Statistics 92(3), 684-689.

Kahneman, D. and R. Sugden (2005). Experienced Utility as a Standard of Policy Evaluation. Environmental and Resource Economics 32(1), 161-181.

Kazemipur, A. (2006). A Canadian Exceptionalism? Trust and Diversity in Canadian Cities. Journal of International Migration and Integration 7(2), 219-240.

Knies, G. and C. K. Spiess (2007). Regional Data in the German Socio-Economic Panel Study (SOEP). DIW Berlin Data Documentation 17.

Lande, R. (1996). Statistics and Partitioning of Species Diversity, and Similarity Among Multiple Communities. Oikos 76, 5-13.

Manacorda, M., A. Manning, and J. Wadsworth (2012). The Impact of Immigration on the Structure of Wages: Theory and Evidence from Britain. Journal of the European Economic Association 10(1), 120-151.

Massey, D. S. and N. A. Denton (1988). The Dimensions of Residential Segregation. Social Forces 67(2), 281-315.

McCulloch, A. (2007). The Changing Structure of Ethnic Diversity and Segregation in England, 1991-2001. Environment and Planning A 39(4), 909-927.

McDonald, D. G. and J. Dimmick (2003). The Conceptualization and Measurement of Diversity. Communication Research 30(1), 60-79.

OECD (2015). International Migration Outlook 2015. OECD Publishing, Paris.

Ottaviano, G. I. and G. Peri (2005). Cities and Cultures. Journal of Urban Economics 58(2), $304-337$.

Ottaviano, G. I. and G. Peri (2006). The Economic Value of Cultural Diversity: Evidence from US Cities. Journal of Economic Geography 6(1), 9-44.

Portes, A. (1998). Social Capital: Its Origins and Applications in Modern Sociology. Annual Review of Sociology 24, 1-24.

Putnam, R. D. (2007). E Pluribus Unum: Diversity and Community in the Twenty-first Century. The 2006 Johan Skytte Prize Lecture. Scandinavian Political Studies 30(2), $137-174$. 
Spolaore, E. and R. Wacziarg (2009). The Diffusion of Development. Quarterly Journal of Economics 124, 469-529.

Spolaore, E. and R. Wacziarg (2016). Ancestry and Development: New Evidence. mimeo.

Stolle, D., S. Petermann, K. Schmid, K. Schönwälder, M. Hewstone, S. Vertovec, T. Schmitt, and J. Heywood (2013). Immigration-related Diversity and Trust in German Cities: The Role of Intergroup Contact. Journal of Elections, Public Opinion \&3 Parties 23(3), 279298.

Sturgis, P., I. Brunton-Smith, J. Kuha, and J. Jackson (2014). Ethnic Diversity, Segregation and the Social Cohesion of Neighbourhoods in London. Ethnic and Racial Studies 37(8), 1286-1309.

Sturgis, P., I. Brunton-Smith, S. Read, and N. Allum (2011). Does Ethnic Diversity Erode Trust? Putnam's "Hunkering Down" Thesis Reconsidered. British Journal of Political Science 41(01), 57-82.

Suedekum, J., K. Wolf, and U. Blien (2014). Cultural Diversity and Local Labour Markets. Regional Studies 48(1), 173-191.

Trax, M., S. Brunow, and J. Suedekum (2015). Cultural Diversity and Plant-level Productivity. Regional Science and Urban Economics 53, 85-96.

van Praag, B. M., P. Frijters, and A. Ferrer-i Carbonell (2003). The Anatomy of Subjective Well-being. Journal of Economic Behavior and Organization 51(1), 29-49.

Winkelmann, L. and R. Winkelmann (1998). Why are the Unemployed so Unhappy? Evidence from Panel Data. Economica 65(257), 1-15.

Winkelmann, R. (2009). Unemployment, Social Capital, and Subjective Well-being. Journal of Happiness Studies 10(4), 421-430. 


\section{Appendix}

Figure A1: Components of Shannon entropy index
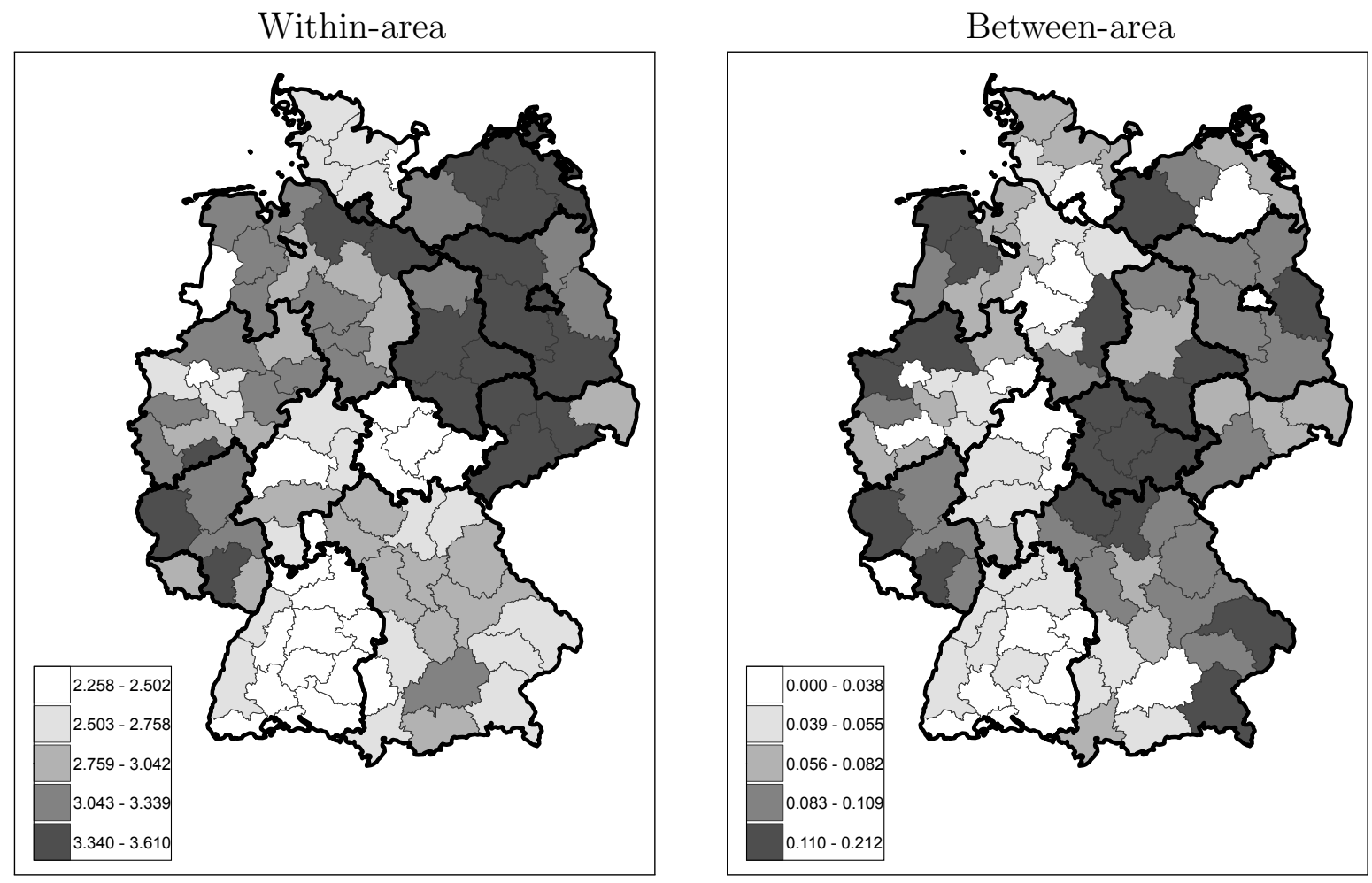

Notes: data refer to averages over 1998 to 2012 . 
Table A1: Multiculturality and happiness - full estimates

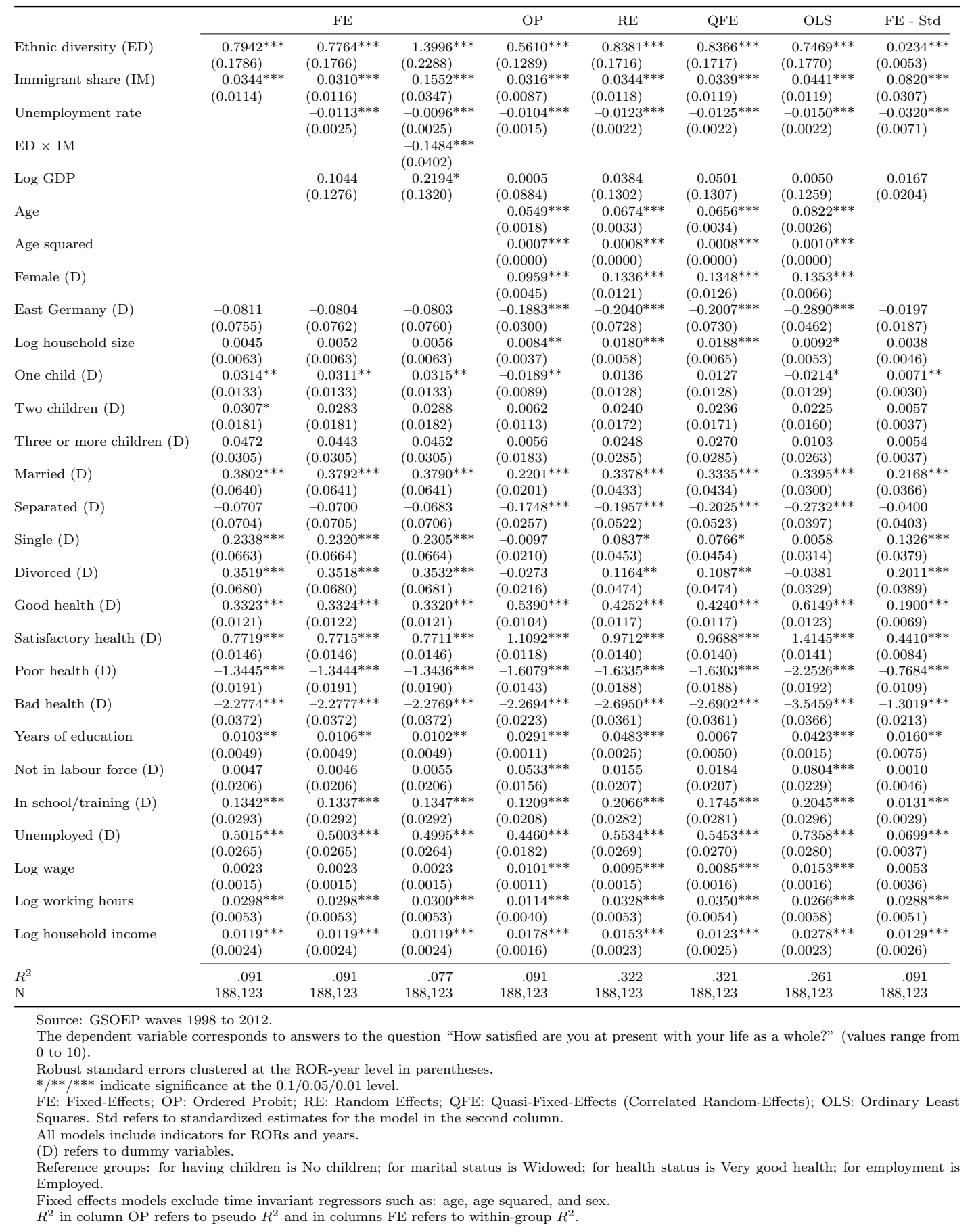


Table A2: Predictions from main effects and interaction models

\begin{tabular}{|c|c|c|c|c|}
\hline $\mathrm{MR} / \mathrm{ED}$ & $1^{s t}$ & $2^{n d}$ & $3^{r d}$ & $4^{t h}$ \\
\hline & & Main eff & ts model & \\
\hline \multirow[t]{2}{*}{$1^{s t}$} & 6.778 & 6.893 & 7.020 & 7.148 \\
\hline & {$[6.431-7.124]$} & {$[6.570-7.215]$} & {$[6.702-7.337]$} & {$[6.810-7.487]$} \\
\hline \multirow[t]{2}{*}{$2^{n d}$} & 6.828 & 6.943 & 7.070 & 7.199 \\
\hline & {$[6.483-7.235]$} & {$[6.622-7.264]$} & $6.754-7.387]$ & {$[6.861-7.537]$} \\
\hline \multirow[t]{2}{*}{$3^{r d}$} & 6.855 & 6.970 & 7.097 & 7.226 \\
\hline & {$[6.510-7.200]$} & {$[6.649-7.292]$} & {$[6.781-7.414]$} & {$[6.888-7.564]$} \\
\hline \multirow[t]{3}{*}{$4^{t h}$} & 6.882 & 6.997 & 7.124 & 7.253 \\
\hline & {$[6.536-7.228]$} & {$[6.676-7.319]$} & {$[6.807-7.441]$} & {$[6.914-7.591]$} \\
\hline & \multicolumn{4}{|c|}{ Intreraction model } \\
\hline \multirow[t]{2}{*}{$1^{s t}$} & 6.831 & 6.927 & 7.057 & 7.156 \\
\hline & {$[6.484-7.179]$} & {$[6.604-7.251]$} & {$[6.738-7.377]$} & {$[6.818-7.495]$} \\
\hline \multirow[t]{2}{*}{$2^{n d}$} & 6.888 & 6.889 & 7.084 & 7.163 \\
\hline & {$[6.541-7.235]$} & {$[6.566-7.211]$} & {$[6.767-7.402]$} & {$[6.824-7.501]$} \\
\hline \multirow[t]{2}{*}{$3^{r d}$} & 6.867 & 6.987 & 7.041 & 7.230 \\
\hline & {$[6.522-7.211]$} & {$[6.665-7.309]$} & {$[6.723-7.360]$} & {$[6.892-7.567]$} \\
\hline \multirow[t]{2}{*}{$4^{t h}$} & 6.924 & 7.096 & 6.982 & 6.846 \\
\hline & {$[6.578-7.271]$} & {$[6.769-7.423]$} & $6.655-7.309]$ & {$[6.437-7.255]$} \\
\hline
\end{tabular}

Source: GSOEP waves 1998 to 2012.

Entries correspond to predictions of the regression in col II (top panel) and col III (bottom panel) of Table 2, conditioning on values of ethnic diversity and immigrant share (mean values of each quartile). Predictions confidence intervals are in brackets. 